\title{
Reduced Dopamine Signaling Impacts Pyramidal Neuron Excitability in Mouse Motor Cortex
}

\author{
Olivia K. Swanson, ${ }^{1,2}$ Rosa Semaan, ${ }^{1}$ and ${ }^{\circledR}$ Arianna Maffei ${ }^{1,2}$
}

https://doi.org/10.1523/ENEURO.0548-19.2021

${ }^{1}$ Department of Neurobiology and Behavior, Stony Brook University, Stony Brook, New York 11794 and ${ }^{2}$ Graduate Program in Neuroscience, Stony Brook University, Stony Brook, New York 11794

\begin{abstract}
Dopaminergic modulation is essential for the control of voluntary movement; however, the role of dopamine in regulating the neural excitability of the primary motor cortex (M1) is not well understood. Here, we investigated two modes by which dopamine influences the input/output function of M1 neurons. To test the direct regulation of M1 neurons by dopamine, we performed whole-cell recordings of excitatory neurons and measured excitability before and after local, acute dopamine receptor blockade. We then determined whether chronic depletion of dopaminergic input to the entire motor circuit, via a mouse model of Parkinson's disease, was sufficient to shift M1 neuron excitability. We show that D1 receptor (D1R) and D2R antagonism altered subthreshold and suprathreshold properties of M1 pyramidal neurons in a layer-specific fashion. The effects of $\mathrm{D} 1 \mathrm{R}$ antagonism were primarily driven by changes to intrinsic properties, while the excitability shifts following D2R antagonism relied on synaptic transmission. In contrast, chronic depletion of dopamine to the motor circuit with 6-hydroxydopamine induced layer-specific synaptic transmission-dependent shifts in M1 neuron excitability that only partially overlapped with the effects of acute D1R antagonism. These results suggest that while acute and chronic changes in dopamine modulate the input/output function of M1 neurons, the mechanisms engaged are distinct depending on the duration and origin of the manipulation. Our study highlights the broad influence of dopamine on M1 excitability by demonstrating the consequences of local and global dopamine depletion on neuronal input/output function.
\end{abstract}

Key words: dopamine; excitability; modulation; motor cortex; neurodegeneration; neuron

\section{Significance Statement}

Dopaminergic signaling is crucial for the control of voluntary movement, and loss of dopaminergic transmission in the motor circuit is thought to underlie motor symptoms in those with Parkinson's disease (PD). Studies in animal models of PD highlight changes in M1 activity following dopamine depletion; however, the mechanisms underlying this phenomenon remain poorly understood. Here we show that diminished dopamine signaling significantly alters the excitability and input/output function of M1 pyramidal neurons. The effects differed depending on the mode and location-local versus across the motor pathway-of the dopamine manipulation. Our results demonstrate how loss of dopamine can engage complex mechanisms to alter M1 neural activity.

\section{Introduction}

Primary motor cortex (M1) exerts powerful control over movement execution through its central location in the motor circuit. It receives inputs from other cortices and

\footnotetext{
Received December 26, 2019; accepted September 7, 2021; First published September 23, 2021.

The authors declare no competing financial interests.
}

the thalamus, the latter relaying converging signals from the basal ganglia and cerebellum (Mink, 1996; BoschBouju et al., 2013; Hooks et al., 2013), and makes direct connections to descending motor tracts (Lemon, 1993).

Author contributions: O.K.S. and A.M. designed research; O.K.S. and R.S. performed research; O.K.S. and R.S. analyzed data; O.K.S. and A.M. wrote the paper. 
Pyramidal neurons in each layer of M1 show distinct projection patterns, with neurons in the superficial layers largely innervating deep layers of M1, other cortices, or striatum; neurons in deep layers primarily project back to the thalamus, striatum, or the corticospinal tract (Oswald et al., 2013). Integral to the transition from input to motor output is neural excitability, which influences the magnitude of the response of a neuron to incoming activity. Excitability can be modulated by motor learning and synaptic plasticity, as well as by changes in overall synaptic drive (Paz et al., 2009; Kida et al., 2016). Neuromodulators further contribute to regulating excitability and input/output functions of neurons. The most crucial neuromodulator for the central control of movement is dopamine.

The influence of dopamine on M1 excitability remains poorly understood (Vitrac and Benoit-Marand, 2017). Anatomical studies report that dopaminergic neurons extend projections to M1 (Fallon and Moore, 1978; Williams and Goldman-Rakic, 1998; Hosp et al., 2011), and dopaminergic activity modulates M1 neurons firing rates (Vitrac et al., 2014). Direct dopaminergic projections are most dense in the deep layers of rodent M1 (Nomura et al., 2014), and D1 receptors (D1Rs) and D2Rs are expressed along the entire depth of the cortical column with laminar-specific density (Ariano and Sibley, 1994; Khan et al., 1998; Lemberger et al., 2007), suggesting possible laminar differences in the effects of local dopamine modulation.

However, the largest dopaminergic input to the motor circuit projects from the substantia nigra pars compacta (SNc) to the basal ganglia (Beckstead et al., 1979), where it signals through D1R and D2R with opposing effects on neural excitability (Surmeier et al., 2007; Azdad et al., 2009; Planert et al., 2013). This, along with its control of synaptic plasticity and transmission, make dopamine a powerful regulator of basal ganglia output (Bagetta et al., 2010) with the ability to affect activity across the motor circuit.

Diminished dopaminergic input to the motor circuit can profoundly impair movement, as observed in Parkinson's disease (PD). While it is clear that depleted dopamine leads to shifts in excitability and synaptic transmission (Blandini et al., 2000; Jankovic, 2008; Grieb et al., 2013; Calabresi and Di Filippo, 2015), this evidence is largely restricted to studies of the basal ganglia. It is unclear if altered basal ganglia activity following nigral dopamine depletion impacts the input/output function of M1 neurons. The classic model of PD postulates that loss of dopamine in the basal ganglia increases the inhibitory output of these nuclei and leads to decreased activation of M1 (Albin et al., 1989; McGregor and Nelson, 2019),

This research was supported by the Thomas Hartman Foundation for Parkinson's Research (Grant 100009356).

Correspondence should be addressed to Arianna Maffei at Arianna. maffei@stonybrook.edu.

https://doi.org/10.1523/ENEURO.0548-19.2021

Copyright @ 2021 Swanson et al.

This is an open-access article distributed under the terms of the Creative Commons Attribution 4.0 International license, which permits unrestricted use, distribution and reproduction in any medium provided that the original work is properly attributed. which could consequently alter excitability. Furthermore, patients with PD exhibit reduced dopaminergic axon density directly in $\mathrm{M} 1$, and functional studies of patients as well as animal models of the disease show altered M1 neural activity, suggesting that depleted dopamine within M1 could also play a role in shifting M1 neural excitability (Gaspar et al., 1991; Lefaucheur, 2005; Lindenbach and Bishop, 2013).

Here, we examined the effect of acute and chronic loss of dopamine signaling on the input/output function of M1 pyramidal neurons using patch-clamp recordings in acute brain slices. First, we tested how acute blockade of D1R and D2R affects neuronal excitability in superficial and deep layers of M1. We then asked whether chronic loss of dopaminergic signaling either in the midbrain or locally within M1 impacts M1 neural excitability. Our results show laminar-specific effects on M1 input/output function through multiple mechanisms. Acute antagonism of D1R and D2R increased excitability through a mix of synaptic transmission dependent and independent mechanisms. Chronic depletion of dopaminergic neurons in the midbrain was also sufficient to engage synapse-dependent modulation of $\mathrm{M} 1$ neurons input/output function, although the effects only partially overlapped with those observed following acute blockade. These data show that loss of dopamine impacts M1 neural excitability and highlights the complex mechanisms that can be engaged depending on laminar location within M1 and specific manipulation of dopamine signaling.

\section{Materials and Methods}

\section{Experimental procedures}

Surgical and experimental procedures followed the guidelines of the National Institutes of Health and were approved by the institutional Animal Care and Use Committee. C57BL/6 mice of both sexes were used in the following experiments. The number of animals and the number of cells in each experimental group were shown as $N$ and $n$, respectively, in the figure legends.

\section{6-Hydroxydopamine injection}

Chronic dopamine depletion was achieved by injection of 6-hydroxydopamine (6OHDA) either in the SNc or in M1. Before surgery, animals received an intraperitoneal injection of desipramine $(1.25 \mathrm{mg} / \mathrm{ml}, 20 \mathrm{ml} / \mathrm{kg})$ to protect noradrenergic and serotonergic afferents from taking up 6OHDA. C57BL/6 mice of both sexes [postnatal day 35 (P35) to P45] were anesthetized $(100 \mathrm{mg} / \mathrm{kg}$ ketamine and $10 \mathrm{mg} / \mathrm{kg}$ xylazine), and a craniotomy was made over the injection site of interest. The 6OHDA solution $(15 \mathrm{mg} / \mathrm{ml}$ in $0.02 \%$ ascorbate) was prepared fresh at the time of injection. Mice unilaterally injected within $\mathrm{M} 1$ received $3 \mu \mathrm{g}$ of 6OHDA or vehicle via two $100 \mathrm{nl}$ injections (bregma, 1.2/ $0.8 \mathrm{~mm}$, midline, $1.1 \mathrm{~mm}$; surface, $0.8 \mathrm{~mm}$ ). Animals unilaterally injected in the SNc received $7.5 \mu \mathrm{g}$ of $6 \mathrm{OHDA}$ or vehicle via two $250 \mathrm{nl}$ injections (bregma, 3.1/2.8; midline, 1.2; surface, 3.93). A pressure injection system (Nanoinject, Drummond) was used for these procedures. Following surgery, animals were monitored daily for food 
and water intake, and administered fluids or softened food when needed.

\section{Cylinder motor task}

Before slice preparation, all 6OHDA or vehicle-injected animals were assessed for motor impairment using the cylinder motor task (lancu et al., 2005). The animal was placed in a clear acrylic cylinder and allowed to freely explore for $10 \mathrm{~min}$ while being filmed with a camera positioned on top of the cylinder. Mirrors were positioned around the cylinder to facilitate visualization of forelimb use and post hoc analysis. Weight-bearing forelimb wall touches were counted over a 3 min period, or a minimum of 20 touches, by an experimenter who was blind to the surgical procedures. Use of the forelimb was quantified as a ratio of wall touches by the forelimb contralateral to the injection (vehicle or 6OHDA) over total wall touches.

\section{Slice electrophysiology}

Animals at P50 to P70, or 2-3 weeks after surgery for the vehicle- and 6OHDA-injected groups, were deeply anesthetized with isoflurane using the bell jar method and rapidly decapitated. Following dissection of the brain, acute $300 \mu \mathrm{m}$ slices containing forelimb M1 (Tennant et al., 2011) were prepared using a vibrating blade microtome (model VT1000S, Leica). Tissue was sectioned in ice-cold oxygenated artificial CSF (ACSF), recovered for $30 \mathrm{~min}$ in $37^{\circ} \mathrm{C} \mathrm{ACSF}$, then allowed to stabilize at room temperature for at least $40 \mathrm{~min}$ before recording. Wholecell patch clamp of visually identified excitatory neurons was performed at room temperature using pulled borosilicate glass pipettes with a resistance of 3-4 M 2 . Dynamic input resistance and frequency-current $(f-l)$ measurements were obtained in current clamp by injecting $700 \mathrm{~ms}$ current steps of increasing intensity ( -100 to $450 \mathrm{pA}$ at $50 \mathrm{pA}$ increments). Action potential threshold and halfwidth were measured on single action potentials at rheobase (rheobase was determined as the current step, in $2 \mathrm{pA}$ increments, that elicited a single action potential). Voltage dependence of the $I_{\mathrm{h}}$ current was measured in current clamp, as the amplitude of the voltage sag current induced by $700 \mathrm{~ms}$ hyperpolarizing current steps from 200 to -25 pA, in 25 pA increments. To block dopamine receptors, either a D1 (SCH23390) or a D2 (sulpiride) receptor antagonist was bath applied for 15 min following a $10 \mathrm{~min}$ baseline. To assess the dependency of dopaminergic activity blockade on synaptic transmission, bath application of D1 or D2 receptor antagonists was repeated in the presence of fast synaptic transmission blockers (APV, DNQX, and picrotoxin). Synaptic transmission blockers were circulated for $10 \mathrm{~min}$ before the application of the dopamine receptor antagonists, and during this time spontaneous activity was monitored in voltage clamp to ensure that all synaptic events onto the recorded cell were abolished. For experiments including bath application of dopamine to provide a dopamine tone to the slice, recordings were performed in the dark to prevent degradation of dopamine. Recorded neurons were exposed to a dopamine solution for 10 min before baseline excitability was measured. After that, the bath application of a solution containing dopamine, $\mathrm{SCH} 23390$, and sulpiride started. Cells were incubated in this cocktail for 15 min before excitability was measured. Series resistance $\left(R_{S}\right)$ was monitored throughout all experiments, and data from cells with $R_{s}>10 \%$ of input resistance or changing $>20 \%$ throughout the recording were excluded from the analysis.

\section{Solutions}

ACSF used in all electrophysiology experiments contained the following (in mM): $126 \mathrm{NaCl}, 3 \mathrm{KCl}, 25 \mathrm{NaHCO}_{3}$, $1 \mathrm{NaHPO}_{4}, 2 \mathrm{MgSO}_{4}, 2 \mathrm{CaCl}_{2}$, and 14 dextrose. The internal solution contained the following (in mM): $100 \mathrm{~K}-\mathrm{Glu}, 20$ $\mathrm{KCl}, 10 \mathrm{~K}$-HEPES, $4 \mathrm{Mg}$-ATP, $0.3 \mathrm{Na}$-GTP, $10 \mathrm{Na}$-phosphocreatine, and $0.4 \%$ biocytin, $\mathrm{pH} 7.35$, titrated with $\mathrm{KOH}$ and adjusted to $295 \mathrm{mOsm}$ with sucrose $\left(\mathrm{E}_{\text {rev }}\left[\mathrm{Cl}^{-}\right]=\right.$ $-49.8 \mathrm{mV}$ ). D1 and D2 receptor antagonists SCH23390 and (S)-(-)-sulpiride (Tocris Bioscience) were prepared in DMSO and diluted in ACSF to a final concentration of 10 $\mu \mathrm{M}$. Solutions containing these antagonists were kept in the dark and bath applied during recording. The baseline ACSF for experiments in which a dopamine tone was provided to the slice contained $10 \mu \mathrm{m}$ dopamine (dopamine hydrochloride, Sigma-Aldrich), $50 \mu \mathrm{m}$ sodium metabisulfite (Sigma-Aldrich), and 0.2\% DMSO, which was added to the solution to balance the DMSO concentration needed to dissolve D1R and D2R blockers. In these experiments, the D1R and D2R blockers $\mathrm{SCH} 23390$ and sulpiride stock solutions in DMSO were added to their final concentration to a fresh, oxygenated volume of the dopamine-ACSF that did not contain DMSO. Experiments performed in the presence of synaptic transmission blockers used ACSF containing the following (in $\mu \mathrm{M}$ ): 20 DNQX, 50 AP5, 20 picrotoxin. The maximum concentration of DMSO for any experimental condition was $0.3 \%$. In experiments using dopamine, there was no net increase in DMSO across conditions. In experiments in which synaptic blockers were added prior and during bath application of D1R or D2R antagonist the transition from pre- to post-dopamine receptor antagonism did not exceed an increase of $0.1 \%$ DMSO.

\section{Immunohistochemistry}

Recorded slices, along with the remaining brain tissue, were postfixed in $4 \%$ paraformaldehyde (0.01 M PBS) $\mathrm{pH} 7.4$, for a minimum of 1 week. Remaining brain tissue containing injection sites was sectioned in the coronal plane at $50 \mu \mathrm{m}$ using a vibrating blade microtome (model VT1000S, Leica) and stored in PBS at $4^{\circ} \mathrm{C}$. Recorded slices were rinsed in PBS and incubated for $30 \mathrm{~min}$ at $45^{\circ} \mathrm{C}$ in an antigen retrieval solution $(10 \mathrm{~mm}$ sodium citrate, $\mathrm{pH}$ 8.5). Slices were again rinsed in PBS, incubated for $2 \mathrm{~h}$ in $50 \mathrm{~mm}$ glycine at room temperature (RT), then following an additional rinse, were preblocked for $3 \mathrm{~h}$ at RT in PBS containing $5 \%$ bovine serum albumin (BSA; Sigma-Aldrich), $5 \%$ normal goat serum (NGS; Vector Laboratories), and $1 \%$ Triton X (Tx; VWR). Slices were then incubated overnight at $4^{\circ} \mathrm{C}$ in an antibody stock solution containing PBS, $1 \%$ BSA, $1 \%$ NGS, $0.1 \% \mathrm{Tx}$, and the following antibodies: streptavidin Alexa Fluor 568 (1:2000; catalog \#S11226, Thermo Fisher Scientific) and mouse anti-GAD67 (1:500; 
catalog \#MAB5406, EMD Millipore). Slices were then rinsed and incubated for $6 \mathrm{~h}$ at RT in the same antibody stock solution, containing goat anti-mouse Alexa Fluor 647 (1:500; catalog \#A-21235, Thermo Fisher Scientific), and were counterstained with Hoechst 33342 stain (1:5000; catalog \#H3570, Thermo Fisher Scientific). Following a final rinse in $0.1 \mathrm{~m}$ phosphate buffer (PB), slices were mounted on gelatin-coated slides and coverslipped with fluorescent mounting medium (Fluoromount-G, Thermo Fisher Scientific).

To assess dopamine neuron or bouton loss, free-floating sections containing injection sites from vehicle or 6OHDA treatments, as well as M1, were rinsed and incubated in antigen retrieval solution and glycine, as described above. Sections were incubated in $0.3 \%$ hydrogen peroxide (Thermo Fisher Scientific) for $30 \mathrm{~min}$ and rinsed, and endogenous avidin and biotin were blocked (Avidin/Biotin Blocking Kit, Vector Laboratories). Following an additional rinse, sections were preblocked in the previously described solution with $0.2 \% \mathrm{Tx}$ for $1 \mathrm{~h}$. Sections were then incubated overnight at $4^{\circ} \mathrm{C}$ in the antibody stock solution with $0.1 \% \mathrm{Tx}$ and rabbit anti-tyrosine hydroxylase $(\mathrm{TH}$; 1:1000; catalog \#ab112, Abcam), rinsed, and incubated for $3 \mathrm{~h}$ at $4^{\circ} \mathrm{C}$ in the antibody solution containing biotinylated goat anti-rabbit (1:200; catalog \#BA-1000, Vector Laboratories). Sections were rinsed and incubated in avidin-biotin horseradish peroxidase (Vectastain Elite $A B C$ Kit, Vector Laboratories) for $1 \mathrm{~h}$ at $\mathrm{RT}$, rinsed, and developed for $60 \mathrm{~s}$ in diaminobenzidine (DAB; Peroxidase Substrate Kit, Vector Laboratories). At the end of this process, sections were rinsed in PB, mounted on gelatincoated slides, and air dried. Slides were then dehydrated in a series of alcohols (70\%, 95\%, 100\%), cleared in xylenes, and coverslipped with Entellan mounting medium.

For determination of cortical layers, a subset of animals was transcardially perfused first with PBS followed by $4 \%$ paraformaldehyde. Brains were dissected and postfixed for $24 \mathrm{~h}$ in $4 \%$ paraformaldehyde then sectioned as described above. The $50 \mu \mathrm{m}$ sections containing M1 were stained with the same methods as above for the expression of the cytoarchitectural marker SMI-32 (1:2000; mouse anti-SMI32, catalog \#801701, BioLegend; 1:500; goat anti-mouse Alexa Fluor 488, catalog \#A-11001, Thermo Fisher Scientific) and counterstained with a pancellular nuclear stain (1:5000; Hoechst 33342 stain; catalog \#H3570, Thermo Fisher Scientific) and neuronal-targeting fluorescent Nissl stain (1:200; Neurotrace 530/615, catalog \#N21482, Thermo Fisher Scientific). Imaging of fluorescently labeled sections was performed on a laser-scanning confocal microscope (FluoView, Olympus), and bright-field images were obtained using a wide-field microscope (Olympus).

\section{Stereological analysis}

The effect of vehicle or 6OHDA on dopaminergic neurons in the SNc and ventral tegmental area (VTA), and on putative dopaminergic boutons in M1 were assessed using unbiased stereological methods with the Stereo Investigator System (MBF Bioscience; Gundersen, 1986; Grieb et al., 2013). These assessments were performed by an experimentalist who was blind both to surgical procedures and electrophysiological results. Sections containing injection sites were processed as described for $\mathrm{TH}$, which labels dopaminergic neurons in the midbrain. Contours of the SNc, VTA, and layers in M1 were traced based on cytoarchitectural bounds from Nisslstained adjacent sections, combined with chemoarchitectonic delineations as previously described (Fu et al., 2012). For counts of $\mathrm{TH}^{+}$neurons in the SNc, eight sections spaced $150 \mu \mathrm{m}$ apart were counted at $400 \times$ magnification, using a grid size of $150 \times 150 \mu \mathrm{m}$ and a $100 \times 100$ $\mu \mathrm{m}$ counting frame. The dissector height was set to $20 \mu \mathrm{m}$ with a guard zone of $2 \mu \mathrm{m}$. Counts of $\mathrm{TH}^{+}$neurons in the VTA were performed in the same manner, with six sections per animal. $\mathrm{TH}^{+}$axon varicosities (putative boutons) were counted in layer $2 / 3$ and layer 5 , across two consecutive $50 \mu \mathrm{m}$ sections at $1000 \times$ magnification, using a grid size of $100 \times 100 \mu \mathrm{m}$ and a $40 \times 40 \mu \mathrm{m}$ counting frame. The dissector height was set to $20 \mu \mathrm{m}$ with a guard zone of $2 \mu \mathrm{m}$. These sampling parameters were sufficient to yield population estimates with a Gunderson coefficient of error of $<10 \%$ for the unlesioned hemispheres and were applied to all cases used in the study. Lesion severity was expressed as a ratio of the estimated population of $\mathrm{TH}^{+}$neurons, or boutons, in the region of interest of the injected hemisphere, relative to that of the contralateral hemisphere. Animals with lesion quantification falling beyond 2 SDs of the mean were excluded.

\section{Data analysis}

Analysis of electrophysiological data was performed with custom-made procedures in Igor (WaveMetrics). Dynamic input resistance was computed as the slope of the current-voltage curve obtained from a series of hyperpolarizing current steps $(-100$ to $0 \mathrm{pA})$. Rheobase was determined by injecting current steps at $2 \mathrm{pA}$ increments until reaching the generation of a single action potential. Action potential threshold and half-width were both calculated at rheobase, in the following manner: (1) the $x$-axis coordinate of the last zero crossing preceding the maximum of the second derivative of the trace was calculated; and (2) this $x$ position was then applied to the original trace, and the threshold was determined to be the $y$ value at this point. To calculate action potential half-width, the action potential amplitude was calculated as the difference in membrane potential between the peak of the action potential and the threshold, then the half-width was calculated as the duration of the action potential at the voltage halfway between action potential threshold and peak amplitude. $f-l$ curves were computed as the average frequency of action potentials for a given current step across all cells in each experimental group (50-450 pA). Voltage dependence of $I_{\mathrm{h}}$ was measured as the difference in membrane potential between absolute minima of the membrane potential within the first $300 \mathrm{~ms}$ of the current step and the average of the steady-state portion of the current step (the last $200 \mathrm{~ms}$ ) across hyperpolarizing steps.

\section{Data presentation and statistical analysis}

Data were compiled and analyzed in Microsoft Excel and the add-in statistical program XLSTAT. Data obtained 
Table 1: Summary statistics table for L2/3 neurons across all experiments

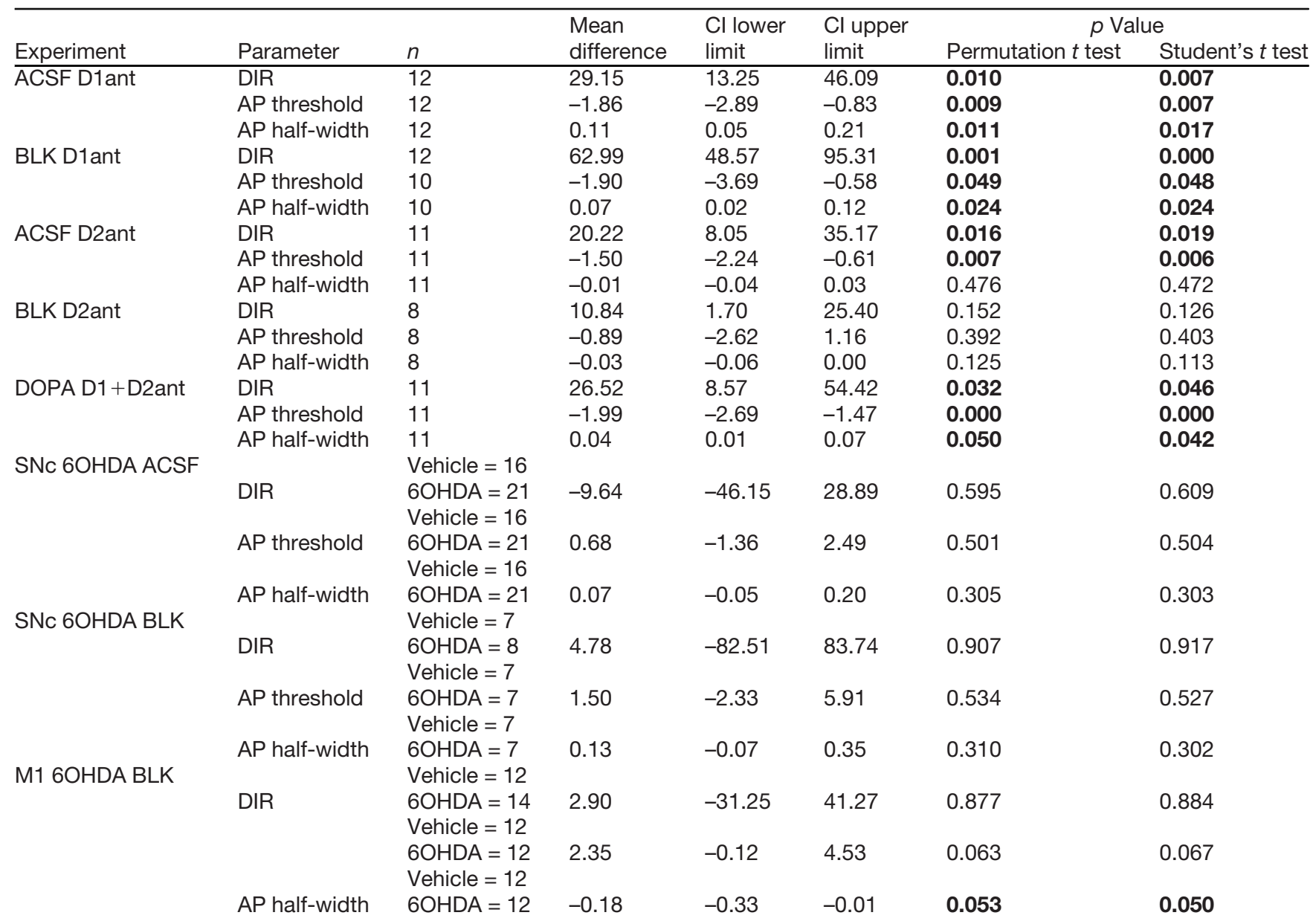

Permutation $t$ test $p$ values are listed alongside Student's $t$ test $p$ values for comparison. Bold $p$ values indicate a $p$ value $\leq 0.05$. AP, Action potential; DIR, dynamic input resistance.

from the cylinder motor assessment and stereological counts are presented as the mean \pm SEM for the number of animals $(N)$ indicated. Voltage dependence on $I_{\mathrm{h}}$ and $f-I$ curve data is represented as line plots where each data point is the mean \pm SEM of the pooled number of neurons $(n)$ across animals $(N)$ indicated in the legend. All other electrophysiology data were imported into https://www. estimationstats.com/ to formulate graphs. Data were tested for normal distribution using the Shapiro-Wilk test. Null hypothesis significance testing between groups was performed using two-tailed unpaired or paired Student's $t$ test or the nonparametric Wilcoxon signed-rank test (for paired data) or Mann-Whitney $U$ test (for unpaired data) for data that did not follow a normal distribution (voltage dependence of $I_{\mathrm{h}}, f-l$ curves). $p$ Values $\leq 0.05$ were considered significant. Where appropriate, we reinforced these analyses using estimation statistics: https://www.estimationstats.com/ was used to import raw data and obtain $95 \%$ confidence intervals (Cls) around the mean difference between groups. Biascorrected and accelerated bootstrap resampling was used to generate 5000 resamples, their distribution, and to construct the $95 \% \mathrm{Cl}$ of the effect size. Individual neurons in drug wash-on experiments are shown as lines as well as the mean \pm SEM in modified Cumming estimation plots (Calin-Jageman and Cumming, 2019; Ho et al., 2019). Individual neurons in lesion experiments are shown as swarm plots and the mean \pm SEM in modified Cumming estimation plots. Alongside the individual neuron data are black dots showing the mean difference between groups (effect size), vertical bars displaying the $95 \% \mathrm{Cls}$, and the underlying resample distribution. Permutation tests were used to determine the likelihood of observing the calculated effect size if the null hypothesis of zero difference was true, and $p \leq 0.05$ was considered significant. The $p$ values for all of the experimental conditions and statistical analyses are reported in Table 1 Layer 2/3 (or L2/3) and Table 2 (L5).

\section{Results}

We performed whole-cell recordings of excitatory neurons in the superficial and deep layers of the forelimb region of M1 (Fig. 1A) to assess the effect of impaired dopamine signaling locally and/or across the motor circuit on neuronal input/output functions. Recorded neurons included in this study showed pyramidal morphology and were negative for GAD67 immunoreactivity (Fig. 1B). To determine laminar borders, sections containing M1 were 
Table 2: Summary statistics table for L5 neurons across all experiments

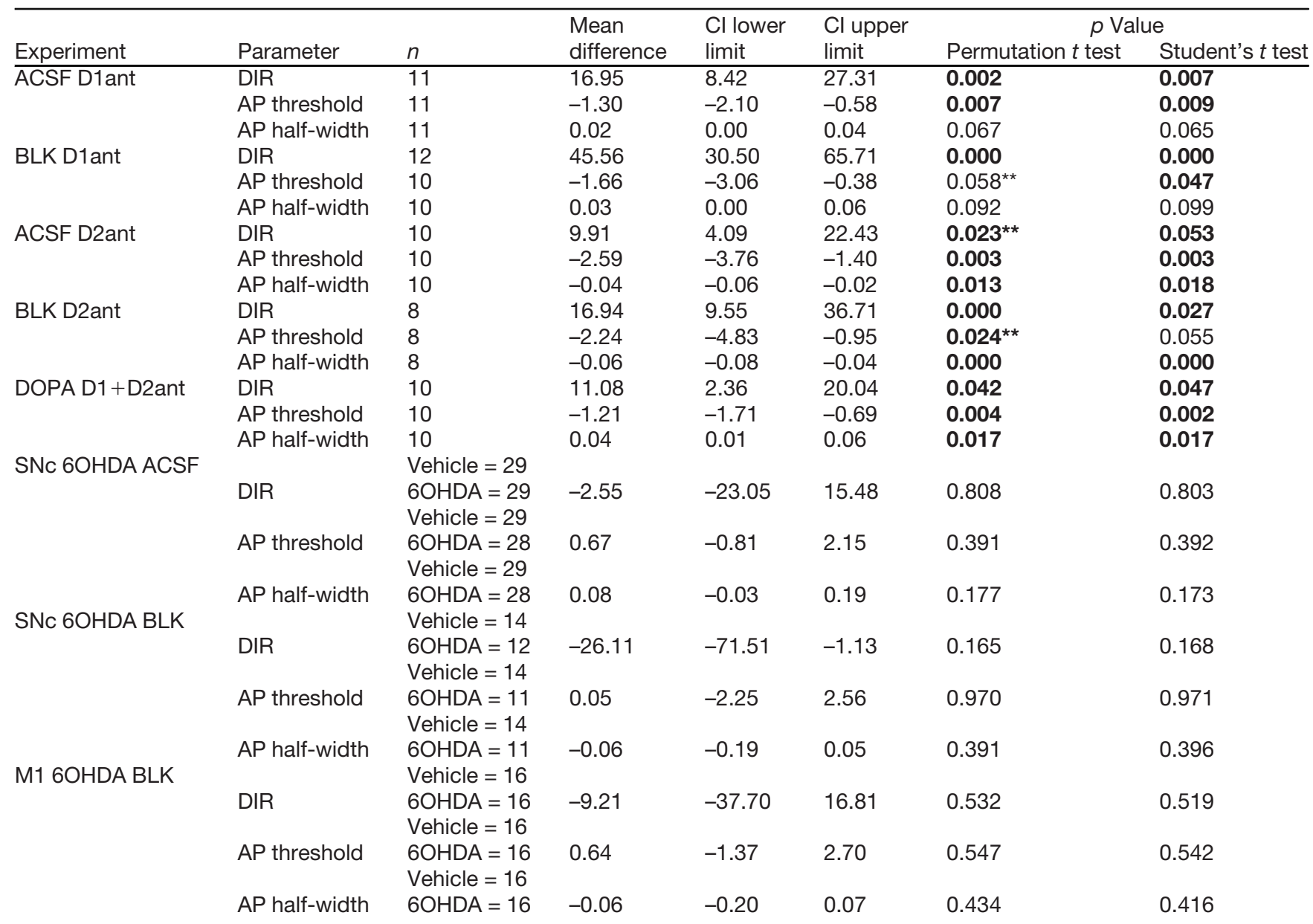

Permutation $t$ test $p$ values are listed alongside Student's $t$ test $p$ values for comparison. Bold $p$ values indicate $p \leq 0.05$. AP, Action potential; DIR, dynamic input resistance.

**Instances where one statistics test was over/under the $0.05 p$ value threshold when the other was not.

stained for the cytoarchitectural marker SMI-32, a nuclear counterstain (Hoechst 33342 stain), and a fluorescent Nissl (Fig. 1C). The top border of Layer 2/3 (L2/3) was placed where cell density sharply drops off as you move toward the pial surface. The bottom of L2/3 was defined at the depth where the cortex transitions from small, densely packed neurons to very large, sparser pyramidal neurons, as visualized with Nissl staining. SMI-32 labels a subset of pyramidal neurons in L2/3 and L5 (Campbell and Morrison, 1989; Voelker et al., 2004), and in dysgranular and agranular cortex it is expressed most strongly in L5 (Barbas and García-Cabezas, 2016). Staining for SMI32 was used to confirm the previously defined laminar borders, and to demarcate the end of $L 5$ and the beginning of L6. Cells were localized to L2/3 or L5 by determining their depth from the cortical surface with post hoc immunostaining of recorded neurons.

\section{Acute blockade of dopamine receptors increases M1 neural excitability}

$\mathrm{D} 1 \mathrm{R}$ and D2R in the rodent brain are expressed in neurons across the cortical mantle, although their expressions show laminar preference (Lemberger et al., 2007; Santana et al., 2009). While dopaminergic modulation through these receptors influences activity in M1 (Molina-Luna et al., 2009; Hosp et al., 2011), the mechanisms underlying the regulation of M1 neurons by dopamine are not clear. To assess how $D 1 R$ and D2R modulate the input/output function of M1 neurons, we first studied the effects of acute dopamine receptor blockade using pharmacological antagonists. We compared current-clamp responses to subthreshold and suprathreshold current steps before and after bath application of dopamine receptor antagonists. To determine whether the modulation of pyramidal neuron input/output function by dopamine receptors is because of intrinsic conductance or synaptic activity, we compared the effect of dopamine receptor antagonists in ACSF, in which spontaneous synaptic activity is present, and in the presence of ionotropic GABA and glutamate receptor blockers.

In a first set of experiments, we examined the effects of the D1R antagonist (D1ant) SCH23390 $(10 \mu \mathrm{m})$ on the excitability (for detailed description of analysis, see Materials and Methods) of pyramidal neurons in L2/3 and L5 of M1 (Fig. 2). In ACSF, bath application of SCH23390 increased the dynamic input resistance of both L2/3 and 
A

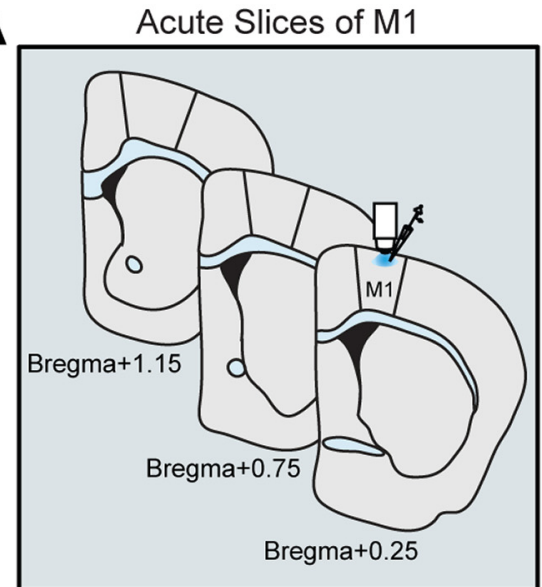

B

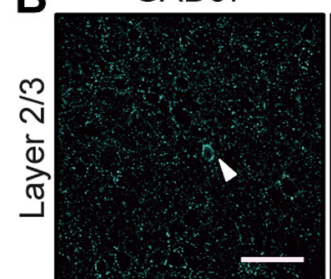

Merge
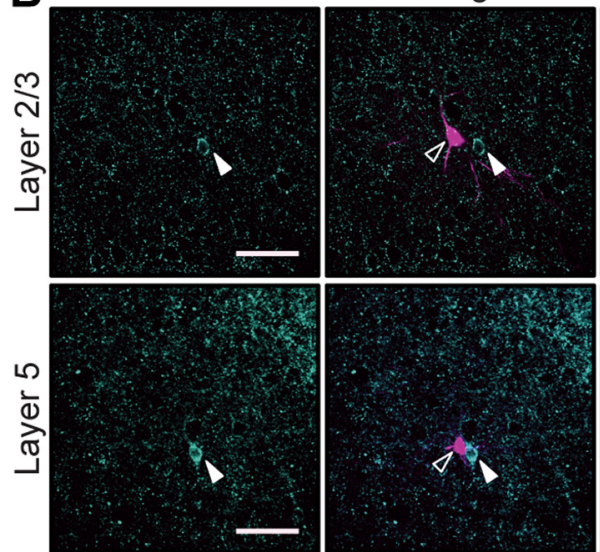

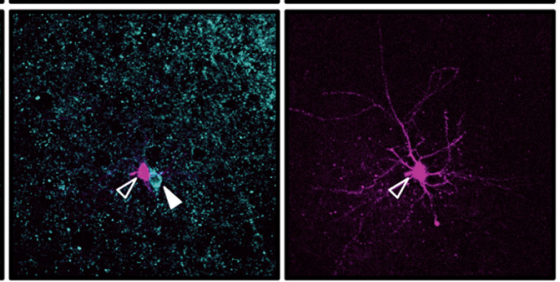

C

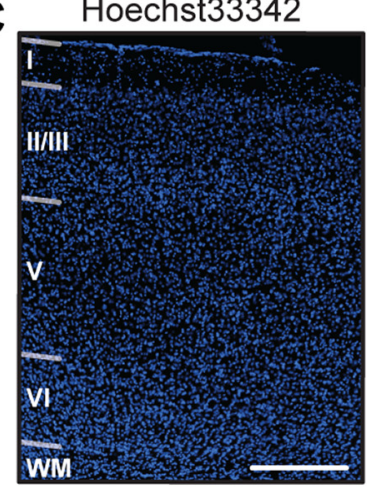

Neurotrace

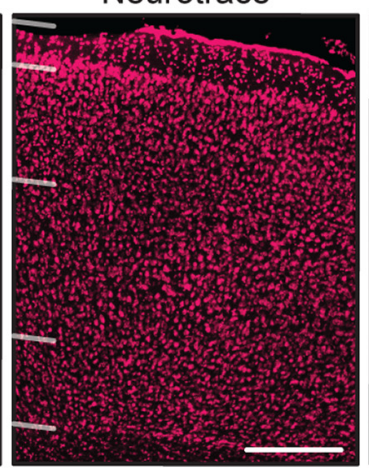

SMI-32

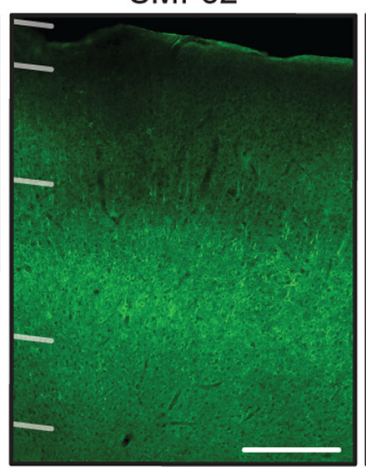

Layer Delineation

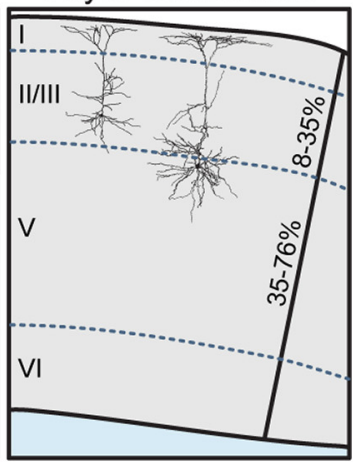

Figure 1. Whole-cell recordings of excitatory neurons in forelimb M1 were localized to $L 2 / 3$ and $L 5$. $\boldsymbol{A}$, Schematic showing the anterior-posterior span of recorded slices, restricted to the forelimb area of M1. B, Recorded neurons were visualized with streptavidin labeling of biocytin and confirmed as excitatory by negative immunoreactivity for GAD67. GAD67 and merged images are shown at one z-plane depth; biocytin images are shown as a collapsed stack spanning the entire neuron. Open arrows, GAD67 biocytin-filled neurons; closed arrows, neighboring GAD67 ${ }^{+}$interneurons (not recorded) at the same depth. Scale bar, $50 \mu \mathrm{m}$. C, Histologic staining of cytoarchitecture used to define cortical layers. Hoechst 33342 stain is a nuclear counterstain of all cells in the region, Neurotrace was used as a neuron-specific stain for somata, SMI-32 labels a subset of pyramidal neurons in layer 3 and layer 5 . Scale bar, $200 \mu \mathrm{m}$. Right-most panel, Two example neurons localized to L2/3 and L5; neurons localized within 8-35\% of the total cortical depth were defined as L2/3; neurons within 35-76\% of cortical depth were defined as L5.

L5 neurons [Fig. 2B; in M $\Omega$ : L2/3 ACSF, $141.32 \pm 9.97$; L2/3 D1ant, $170.49 \pm 12.24(p=0.007) ;$ L5 ACSF, $106.33 \pm 14.80$; L5 D1ant, $123.28 \pm 15.66(p=0.007)]$. This effect was potentiated in the presence of ionotropic GABA and glutamate receptor blockers (BLK), suggesting that increased dynamic input resistance induced by D1R blockade occurs independent of fast synaptic transmission [Fig. $2 B$; in $M \Omega$ : L2/3 BLK, 139.06 \pm 12.04; L2/3 D1ant, 201.73 $\pm 20.41(p=0.001)$; L5 BLK, $91.79 \pm 11.29$; L5 D1ant, $136.25 \pm 20.90(p=0.002)]$.

In response to hyperpolarizing current steps, neurons showed a voltage sag during the initial portion of the response, which is typically associated with the presence of hyperpolarization-activated cation channels $(\mathrm{HCNs})$ mediating $I_{\mathrm{h}}$ (Rosenkranz and Johnston, 2006; Hogan and Poroli, 2008). Bath application of SCH23390 increased the amplitude of the voltage sag in both L2/3 and L5 neurons, and this change persisted in the presence of synaptic blockers, confirming the independence of this effect from fast synaptic transmission (Fig. 2C).

Next, we assess the effect of D1R blockade on the suprathreshold portion of the input/output function. We first compared action potential properties at rheobase: the action potential threshold was more hyperpolarized in both L2/3 and L5 neurons in the presence of SCH23390 [Fig. 2D; in $\mathrm{mV}$ : L2/3 ACSF, $-44.10 \pm 1.26$; L2/3 D1ant, $-45.96 \pm$ $1.28(p=0.0073) ;$ L5 ACSF, $-44.57 \pm 0.84$; L5 D1ant, $-45.87 \pm 0.93(p=0.0087)]$. When this experiment was repeated in the presence of fast synaptic transmission blockers, the effect remained in both layers [Fig. 2D; in $\mathrm{mV}$ : L2/3 BLK, $-41.57 \pm 0.93$; L2/3 D1ant, $-43.47 \pm$ $0.88(p=0.048) ;$ L5 BLK, $-43.49 \pm 1.25$; L5 D1ant, $-45.14 \pm 1.63(p=0.047)]$. Blocking D1Rs also increased action potential half-width in $\mathrm{L} 2 / 3$, but not in $\mathrm{L} 5$ neurons [Fig. 2E; in ms: L2/3 ACSF, $1.63 \pm 0.071$; L2/3 D1ant, $1.74 \pm 0.091(p=0.017)$; L5 ACSF, $1.32 \pm 0.077$; L5 D1ant, $1.35 \pm 0.075(p=0.065)]$. This effect of D1R blockade on L2/3 neuron half-width persisted in synaptic transmission blockers [Fig. 2E; in ms: L2/3 BLK, 1.43 \pm 0.041; L2/3 D1ant, $1.50 \pm 0.059(p=0.024) ;$ L5 BLK, $1.31 \pm$ 0.053; L5 D1ant, $1.34 \pm 0.058(p=0.099)]$. Thus, dopamine affects action potential properties of M1 pyramidal neurons by acting through distinct mechanisms in superficial and deep layers. 
A

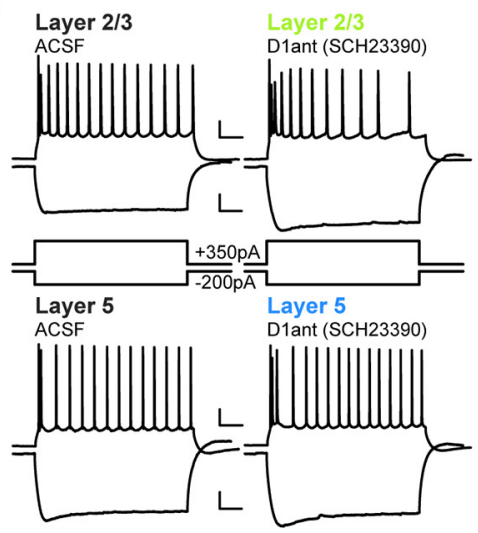

D
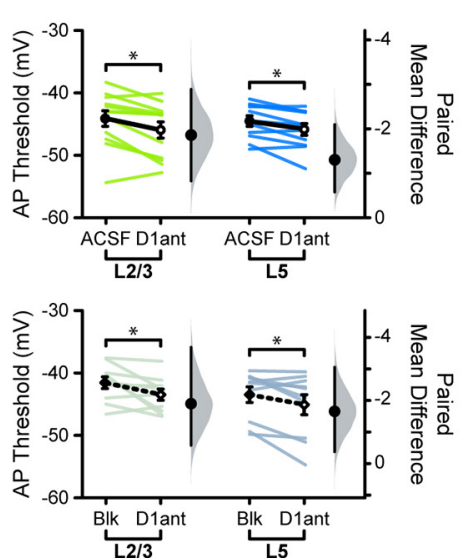

B
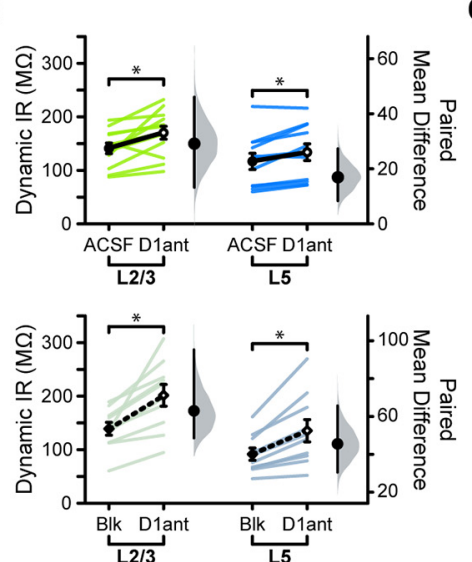

E
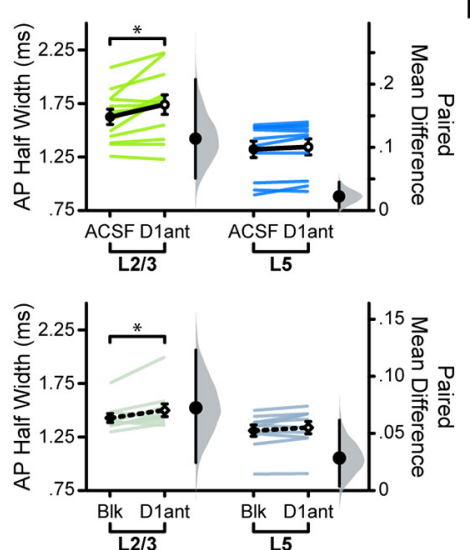

C
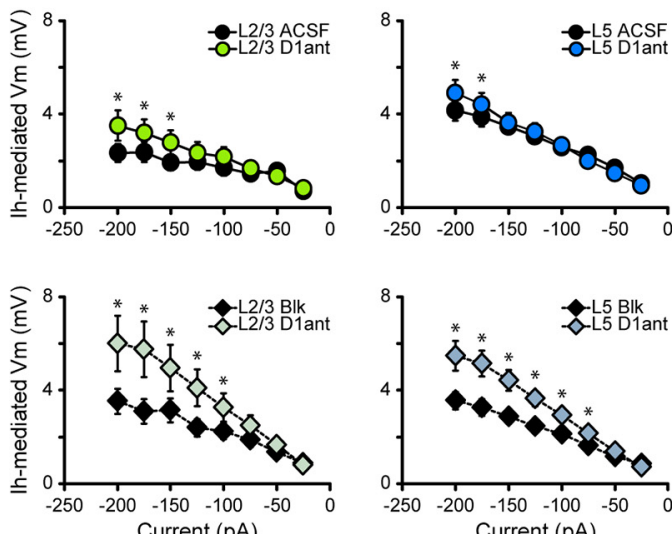

$\mathbf{F}$
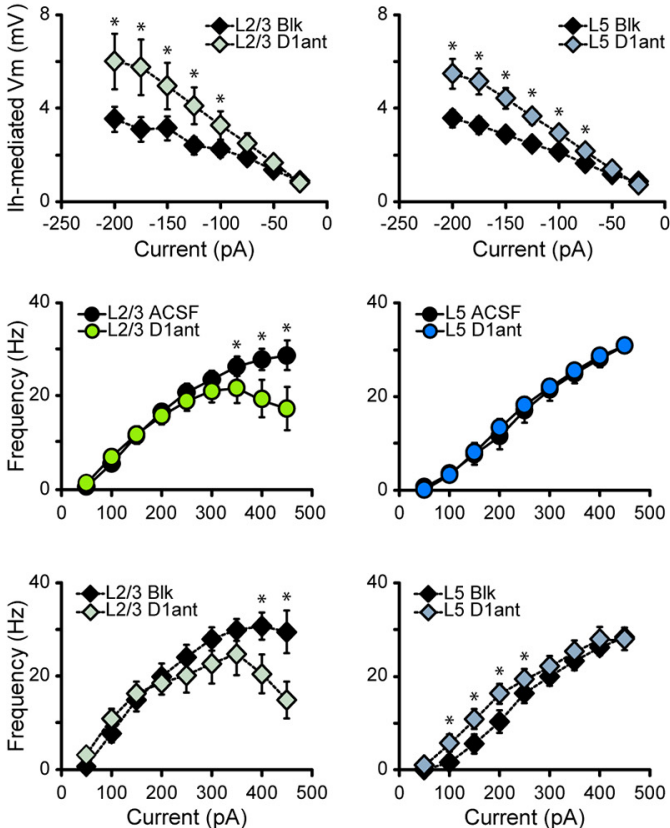

Figure 2. Acute D1R blockade shifts excitability of M1 neurons. $\boldsymbol{A}$, Superimposed responses to hyperpolarizing and depolarizing current steps in individual L2/3 and L5 neurons before and after bath application of D1R antagonist SCH23390 (D1ant, $10 \mu \mathrm{M}$ ). Scale bar: Top, $20 \mathrm{mV}, 100 \mathrm{~ms}$; bottom: $10 \mathrm{mV}, 100 \mathrm{~ms}$. $\boldsymbol{B}-\boldsymbol{F}$. Summary excitability plots for excitatory neurons in L2/3 (green) and L5 (blue) before and after D1ant application, in baseline (ACSF) or synaptic blocker (BLK: $20 \mu \mathrm{m}$ picrotoxin, $20 \mu \mathrm{m}$ DNQX, $50 \mu \mathrm{m}$ AP5) conditions. Modified Cumming plots show raw data of individual neurons as lines, overlayed with the mean \pm SEM. To the right of each group of raw data are the effect size (black circle), corresponding $95 \%$ Cls (black vertical bars), and the underlying bootstrap sampling distribution. $\boldsymbol{B}$, Dynamic input resistance across hyperpolarizing current steps. C, Voltage dependence of $I_{\mathrm{h}}$ mediated voltage sag elicited by hyperpolarizing current. $\boldsymbol{D}$, Action potential threshold at rheobase. $\boldsymbol{E}$, Action potential half-width at rheobase. $\boldsymbol{F}$, Action potential frequency during suprathreshold current injections (ACSF L2/3 neurons: $N=6, n=12$; ACSF L5 neurons: $N=6, n=11$; BLK L2/3 neurons: $N=5, n=10$; BLK L5 neurons: $N=6, n=10$. Data are shown as the mean \pm SEM. ${ }^{*} p \leq 0.05$.

Bath application of the D1R antagonist SCH23390 unveiled laminar differences in the effects of acute D1R blockade on the input/output curve. Comparison of relationships between action potential frequency and injected current ( $f-l$ curve) in ACSF and acute D1R blockade showed that the ability of L2/3 neurons to increase their firing rates in response to increasing current steps was impaired, significantly reducing the maximum firing rate. In contrast, $\mathrm{SCH} 23390$ did not affect the $f-I$ curve of $L 5$ neurons (Fig. 2F). The changes in the $f-l$ curve of $L 2 / 3$ neurons persisted in synaptic blockers, indicating that they depend on the modulation of voltage-gated conductance. Interestingly, in L5, pharmacological blockade of ionotropic synaptic receptors unveiled a previously masked effect of D1R blockade on the $f-l$ curve: an increase in firing rate selectively in the linear portion of the $f-l$ curve, the range in which firing rates of neurons show high sensitivity to small changes in current injection. The increase in input resistance and hyperpolarization of action potential threshold points to a net increase in M1 pyramidal neuron excitability in the absence of dopamine activation of D1R. In L2/3, selectively blocking dopamine signaling through $\mathrm{D} 1 \mathrm{R}$ also results in a decreased maximum firing rate, suggesting that large incoming input would result in reduced output.

Dopaminergic modulation of neuronal activity can also rely on D2Rs, which are expressed in $\mathrm{M} 1$ neurons (Santana et al., 2009). Previous in vivo studies reported that the activation of D2Rs increases the firing rate of M1 pyramidal neurons in anesthetized animals and can alter motor maps, but the mechanisms underlying these effects are unclear (Hosp et al., 2009; Vitrac et al., 2014). To assess whether D2Rs modulate the input/output curve of M1 pyramidal neurons, we repeated the experiments above using the D2R antagonist (D2ant) sulpiride (10 $\mu \mathrm{M}$; Fig. 3). Bath application of sulpiride increased the input resistance of $L 2 / 3$ and $L 5$ neurons [Fig. $3 B$; in $\mathrm{M} \Omega$ : L2/3 ACSF, $108.56 \pm 11.01$; L2/3 
A
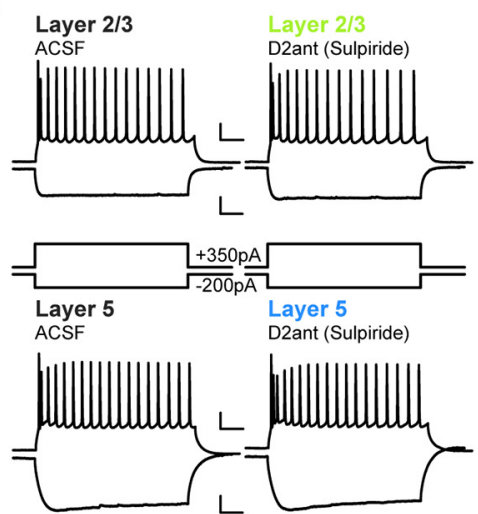

D
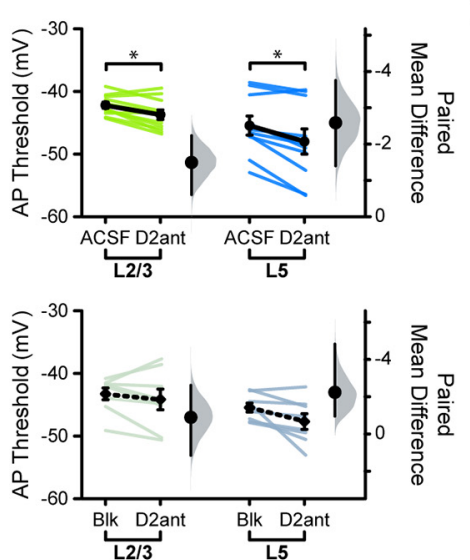

B
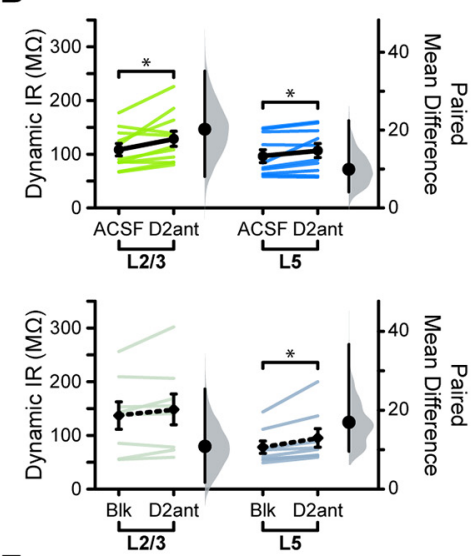

E
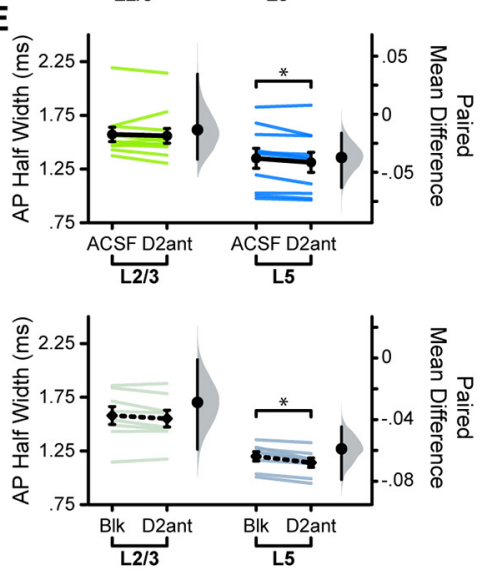

C
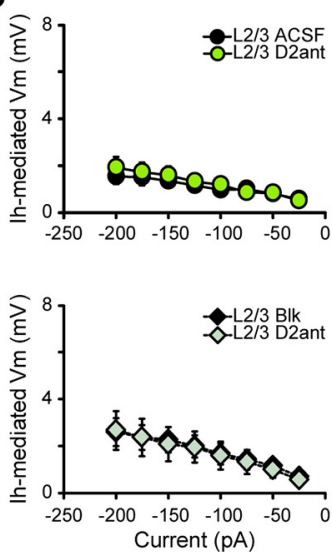

F
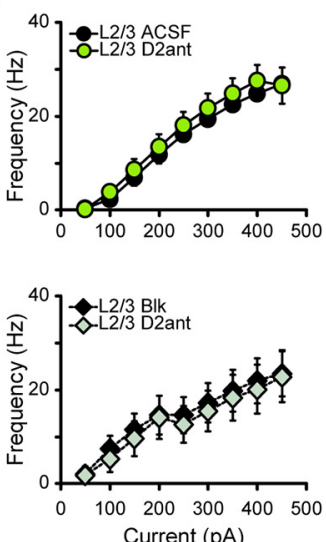
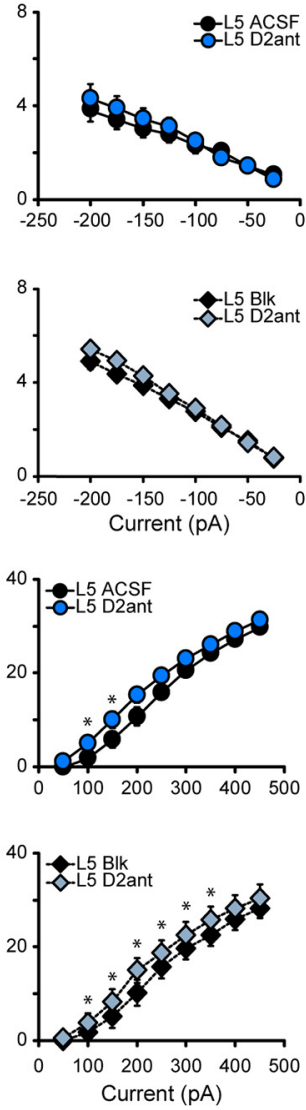

Figure 3. Acute D2R blockade shifts excitability of M1 neurons. A, Superimposed responses to hyperpolarizing and depolarizing current steps in individual L2/3 (green) and L5 (blue) neurons before and after bath application of D2R antagonist sulpiride (D2ant, $10 \mu \mathrm{m}$ ) Scale bar: Top, $20 \mathrm{mV}, 100 \mathrm{~ms}$; bottom, $10 \mathrm{mV}, 100 \mathrm{~ms}$. $\boldsymbol{B}-\boldsymbol{F}$, Summary excitability plots for excitatory neurons in L2/3 and L5 before and after D2ant application, in baseline (ACSF) or synaptic blocker (BLK: $20 \mu \mathrm{m}$ picrotoxin, $20 \mu \mathrm{m}$ DNQX, $50 \mu \mathrm{m}$ AP5) conditions. Modified Cumming plots show raw data of individual neurons as lines, overlayed with the mean $\pm \mathrm{SEM}$. To the right of each group of raw data are the effect size (black circle), corresponding 95\% Cls (black vertical bars), and the underlying bootstrap sampling distribution. $\boldsymbol{B}$, Dynamic input resistance across hyperpolarizing current steps. $\boldsymbol{C}$, Voltage dependence of $I_{\mathrm{h}}$-mediated voltage sag elicited by hyperpolarizing current. $\boldsymbol{D}$, Action potential threshold at rheobase. $\boldsymbol{E}$, Action potential half-width at rheobase. $\boldsymbol{F}$, Action potential frequency during suprathreshold current injections. ACSF L2/3 neurons: $N=5, n=11$; ACSF L5 neurons: $N=5$, $n=10$; Blk L2/3 neurons: $N=4, n=8$; Blk L5 neurons: $N=4, n=8$. Data are shown as mean \pm SEM. ${ }^{*} p \leq 0.05$.

D2ant, $127.78 \pm 14.12(p=0.019) ;$ L5 ACSF, $97.1763 \pm$ 11.94; L5 D2ant, $107.09 \pm 12.69(p=0.053)]$. The increase in input in L2/3 neurons was eliminated by $\mathrm{GABA}_{A}$, AMPA, and NMDA receptor antagonists, suggesting that it relies on the modulation of synaptic transmission. In contrast, the application of sulpiride in L5 in the presence of synaptic receptor blockers amplified the increase in input resistance [Fig. $3 B$; in $\mathrm{M} \Omega$ : L2/3 BLK, $137.33 \pm 25.23$; L2/3 D2ant, $148.17 \pm 28.67$ $(p=0.13) ; \quad$ L5 BLK, 78.69 $\pm 11.69 ; \quad$ L5 D2ant, $95.63 \pm$ $17.39(p=0.027)]$. Sulpiride did not affect the voltage sag in either L2/3 or L5 pyramidal neurons (Fig. 3C), suggesting that D2Rs do not modulate this current in M1.

Bath application of sulpiride hyperpolarized the action potential threshold [Fig. 3D; in mV: L2/3 ACSF, $-42.22 \pm$ 0.49 ; L2/3 D2ant, $-43.72 \pm 0.75(p=0.0063)$; L5 ACSF, $-45.46 \pm 1.51$; L5 D2ant, $-48.05 \pm 2.00(p=0.003)]$. In both layers, this effect was eliminated by the presence of ionotropic GABA and glutamate receptor blockers [Fig. $3 D$; in $\mathrm{mV}$ : L2/3 BLK, $-43.26 \pm 0.98$; L2/3 D2ant, $-44.15 \pm 1.68 \quad(p=0.41) ; \quad$ L5 BLK, $-45.48 \pm 0.75 ; \quad$ L5 D2ant, $-47.72 \pm 1.25(p=0.055)]$. Sulpiride had no effect on action potential half-width of L2/3 neurons, but it did decrease the half-width of L5 cells in both ACSF and synaptic blockers [Fig. $3 E$; in ms: L2/3 ACSF, $1.57 \pm 0.067$; L2/3 D2ant, $1.56 \pm 0.069 \quad(p=0.47) ; \quad$ L5 ACSF, $1.35 \pm$ 0.093; L5 D2ant, $1.31 \pm 0.093(p=0.018) ;$ L2/3 BLK, $1.58 \pm 0.083 ; \quad L 2 / 3 \quad$ D2ant, $1.55 \pm 0.078 \quad(p=0.11) ; \quad$ L5 BLK, $1.20 \pm 0.043 ; \quad$ L5 D2ant, $1.14 \pm 0.043 \quad(p=4.28 \times$ $\left.\left.10^{-4}\right)\right]$. This effect in L5 opposes that of D1R antagonism, suggesting that $\mathrm{D} 2 \mathrm{R}$ antagonism plays a unique role in modulating action potential properties in M1. Finally, sulpiride had no effect on the $f-l$ curve of $L 2 / 3$ pyramidal neurons. However, in L5 it increased the action potential frequency in the linear portion of the $f-l$ curve, increasing L5 neuron output to small changes in input current. The effect of sulpiride on the $f-l$ curve of $L 5$ neurons persisted 
A
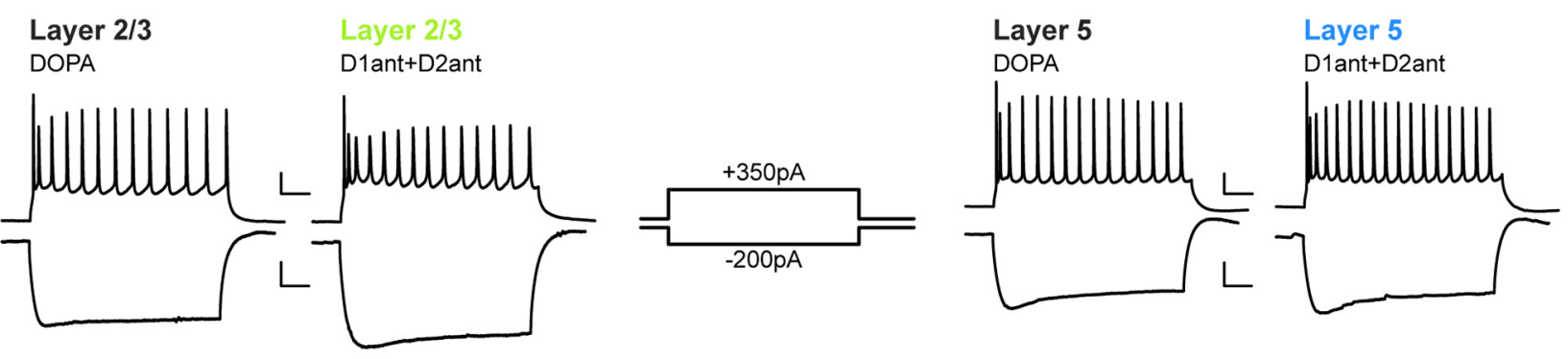

B

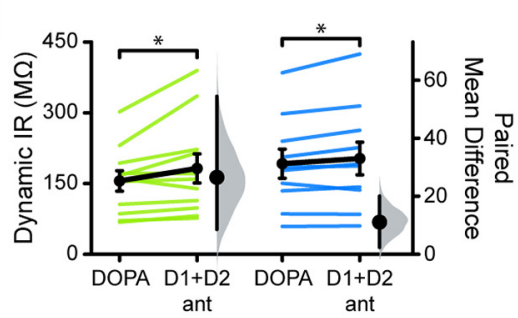

C

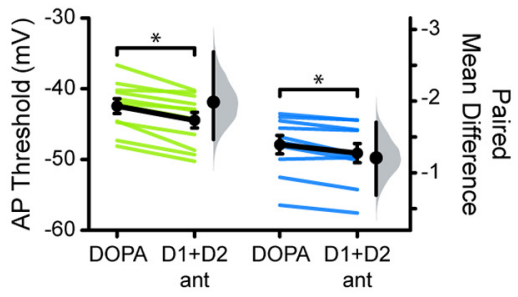

D

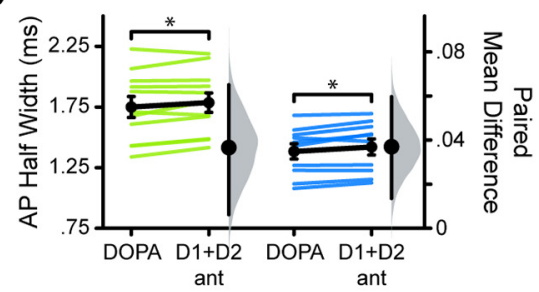

E

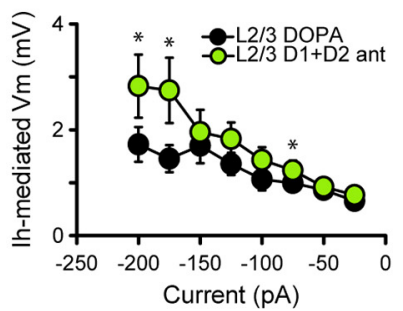

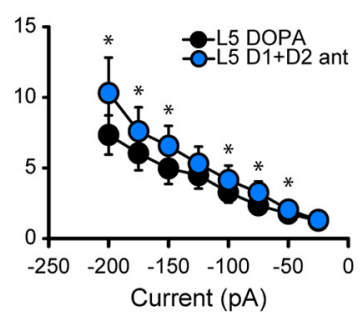

$\mathbf{F}$

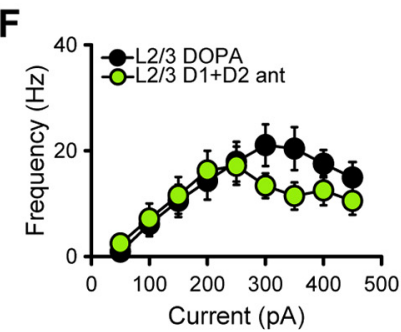

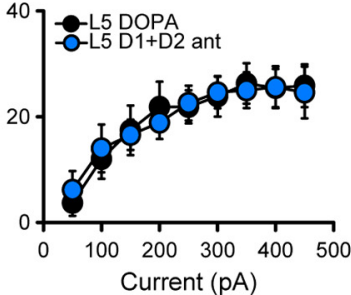

Figure 4. Combined $D 1 R+D 2 R$ blockade in dopamine-primed slices of $M 1$ recapitulates the results of individual antagonist experiments. $\boldsymbol{A}$, Superimposed responses to hyperpolarizing and depolarizing current steps in individual L2/3 (green) and L5 (blue) neurons before and after bath application of SCH23390 (D1ant) and Sulpiride (D2ant). Scale bar: Top, $20 \mathrm{mV}, 100 \mathrm{~ms}$; bottom, $10 \mathrm{mV}$, $100 \mathrm{~ms}$. B-F, Summary excitability plots for excitatory neurons in L2/3 and L5 before and after D1ant+D2ant application, in dopamine $(10 \mu \mathrm{M})$ ACSF. Modified Cumming plots show raw data of individual neurons as lines, overlayed with the mean \pm SEM. To the right of each group of raw data are the effect size (black circle), corresponding 95\% Cls (black vertical bars), and the underlying bootstrap sampling distribution. $\boldsymbol{B}$, Dynamic input resistance across hyperpolarizing current steps. $\boldsymbol{C}$, Action potential threshold at rheobase. $\boldsymbol{D}$, Action potential half-width at rheobase. $\boldsymbol{E}$, Voltage dependence of $I_{\mathrm{h}}$-mediated voltage sag elicited by hyperpolarizing current. $\boldsymbol{F}$, Action potential frequency during suprathreshold current injections. DOPA L2/3 neurons: $N=7, n=11$; DOPA L5 neurons: $N=5, n=10$. Data are shown as the mean \pm SEM. ${ }^{*} p \leq 0.05$.

in the presence of synaptic blockers, suggesting that this effect is independent of fast synaptic transmission (Fig. $3 F$ ). Taken together, these data suggest that while D2Rs play a role in M1 excitability, the consequences of an acute loss of D2R activity are more subtle than those of D1R antagonism. In both layers, acute blockade of D2R signaling results in a net increase in excitability. While in L2/3 this effect depends on synaptic transmission, in L5 it is primarily dependent on the modulation of intrinsic conductance.

To provide additional statistical analysis of these results, we performed estimation statistics on input resistance, threshold, and half-width data from dopamine receptor antagonism experiments. These analyses measured effect size (mean difference) between control and test groups and use bootstrap resampling to construct 95\% Cls for each parameter. Permutation $t$ tests were used to assess the likelihood of observing the effect size if the null hypothesis of zero difference were true. Permutation $t$ test $p$ values were consistent with the reported Student's $t$ test values in nearly all instances
(Tables 1 for L2/3 neurons, 2 for L5 neurons). In the three cases where the permutation test results differed in significance from the Student's $t$ test (L5; Table 2, marked with asterisks), the $p$ values hovered around 0.05 . Consistency in both statistical analyses reinforces a physiological role of dopamine receptor activity in $\mathrm{M} 1$ and strengthens the assertion that loss of $D 1$ or $D 2$ receptor function impacts the excitability of neurons in $L 2 / 3$ and $L 5$. These results point to the laminar specificity of dopamine receptor function in M1 and support the hypothesis that dopamine modulates M1 excitability through complex mechanisms involving both intrinsic conductance and synaptic transmission.

\section{Effects of D1R and D2R antagonism persist in the presence of dopamine tone}

Our data indicate that dopaminergic modulation plays a role in maintaining excitability of pyramidal neurons in M1. One consideration when interpreting these results is that while ex vivo brain slices preserve much of the synaptic circuitry, endogenous levels of dopamine in M1 may be 
significantly reduced, and dopamine is highly sensitive to oxidation. Furthermore, both $\mathrm{SCH} 23390$ and sulpiride were dissolved into a DMSO vehicle, which has been shown to impact excitability after long slice incubations (Tamagnini et al., 2014). To address these factors, we primed acute M1 slices with a dopamine-ACSF solution before bath applying dopamine receptor antagonists. In this experiment, the concentration of DMSO remained constant before and after the application of dopamine receptor blockers. Considering that previous results showed that D1R and D2R antagonism drove partially overlapping shifts in M1 neuron excitability, we combined $\mathrm{SCH} 23390$ and sulpiride in the antagonist condition and posited that simultaneous $\mathrm{D} 1 \mathrm{R}$ and $\mathrm{D} 2 \mathrm{R}$ antagonism would yield results similar to those observed with individual antagonists.

Bath application of the combined antagonists, in the presence of dopamine, reproduced our previous results and reinforced the consequence of reduced dopamine signaling on excitability in M1 (Fig. 4). In both L2/3 and L5 neurons, input resistance was increased [Fig. $4 B$; in $M \Omega$ : L2/3 DOPA, $155.62 \pm 21.54$; L2/3 DOPA+D1ant+D2ant, $182.14 \pm 30.86(p=0.046) ;$ L5 DOPA, $192.12 \pm 30.76$; L5 DOPA+D1ant+D2ant, $203.20 \pm 34.73(p=0.047)]$, action potential threshold was hyperpolarized [Fig. $4 C$; in $\mathrm{mV}$ : L2/3 DOPA, $-42.46 \pm 1.04$; L2/3 DOPA+D1ant+D2ant, $-44.45 \pm 1.11(p=0.00011) ;$ L5 DOPA, $-47.89 \pm 1.30$; L5 DOPA+D1ant+D2ant, $-49.10 \pm 1.35(p=0.002)]$, and action potential half-width was increased [Fig. 4D; in ms: L2/ 3 DOPA, $1.75 \pm 0.086$; L2/3 DOPA+D1ant+D2ant, $1.79 \pm$ $0.080(p=0.042) ;$ L5 DOPA, $1.38 \pm 0.063$; L5 DOPA+D1ant+ D2ant, $1.42 \pm 0.066(p=0.017)]$. Similar to D1R antagonism alone, combined D1R/D2R antagonism in the presence of dopamine significantly increased the voltage sag in both L2/3 and L5 neurons (Fig. 4E). However, there was no significant effect on the $f-l$ curve of either L2/3 or L5 neurons, suggesting that simultaneous antagonism of both D1R and D2R may exert a unique effect on the firing rates of neurons in $\mathrm{M} 1$ compared with when only one type is blocked (Fig. 4F). Analyses of these data using an estimation statistics approach bolstered these results: in all cases, permutation $t$ tests of the bootstrap resamples indicate that an acute blockade of D1R and D2R, in the presence of dopamine, drives an increase in the excitability of L2/3 and L5 neurons in M1 (Tables 1, L2/3, 2, L5).

\section{Chronic midbrain dopamine depletion alters M1 neural excitability}

Diminished dopamine signaling in the motor system and progressive motor impairment are hallmarks of PD. Patients, as well as animal models of the disease, exhibit motor cortex dysfunction. We hypothesized that chronic loss of dopaminergic input to the motor circuit alters the excitability of M1 pyramidal neurons, possibly providing a mechanism for impaired motor cortex activity. We asked whether chronic loss of dopaminergic activity across the entire motor circuit, or locally within $\mathrm{M} 1$, is sufficient to shift the excitability of M1 neurons, and recapitulate the results of the acute dopamine receptor blockade
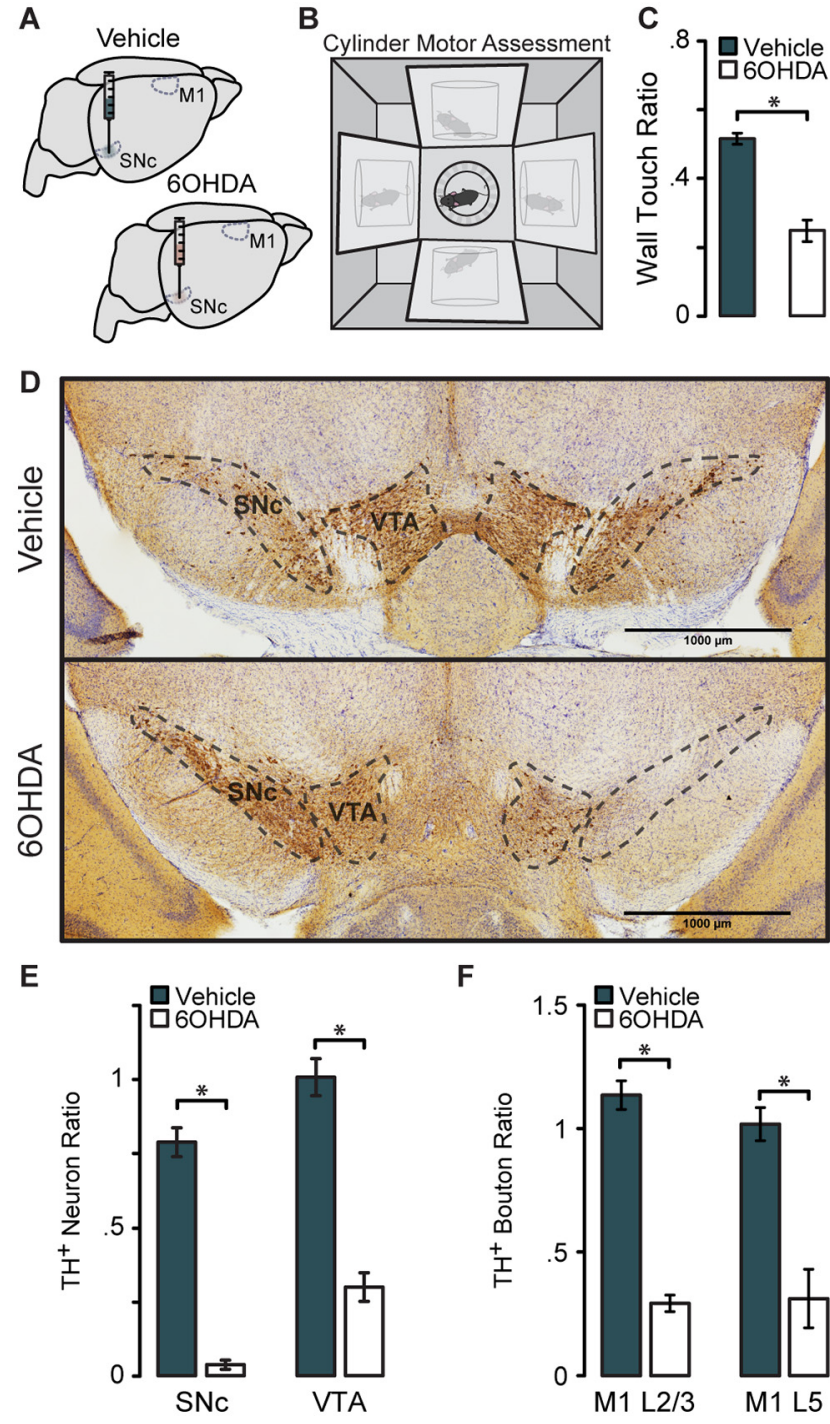

Figure 5. Validation of the 6OHDA model of Parkinson's disease. $\boldsymbol{A}$, Unilateral injection of 6OHDA or vehicle, centered on the SNc. B, Schematic of the cylinder motor assessment. $\boldsymbol{C}$, Quantification of weight-bearing wall touches measured as a ratio of forelimb use contralateral versus ipsilateral to the injected hemisphere. $\boldsymbol{D}$, Immunolabeled $\mathrm{TH}^{+}$dopaminergic neurons visualized with DAB in the SNc and VTA. $\boldsymbol{E}$, Summary of stereological counts of $\mathrm{TH}^{+}$neurons in the SNc and VTA of lesioned or vehicle-injected animals. (vehicle animals, $N=18$; 6 OHDA animals, $N=17$; data are shown as the mean \pm SEM. ${ }^{*} p<0.0001$. $\boldsymbol{F}$, Summary stereological counts of $\mathrm{TH}^{+}$boutons in M1 of a subset of animals. Vehicle animals, $N=2$; 6 OHDA animals, $N=2$. Data are shown as the mean \pm SEM. ${ }^{*} p \leq 0.05$.

experiments. We unilaterally injected 6OHDA or an equivalent volume of vehicle as a control into the midbrain centering the injection site on the SNc (Fig. 5). Dopamine depletion of midbrain dopaminergic neurons with 6OHDA is widely used as a model of PD and is known to induce motor impairment. Two weeks after injection, and just before recording, the movement of each animal was assessed with a cylinder motor task (Fig. 5C). 6OHDAinjected mice showed reduced use of the forelimb 
A

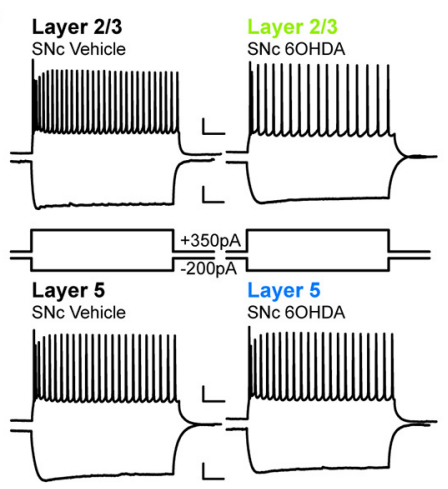

B
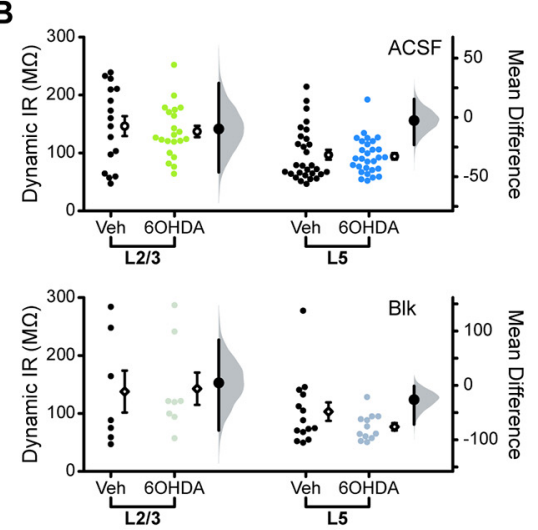

C
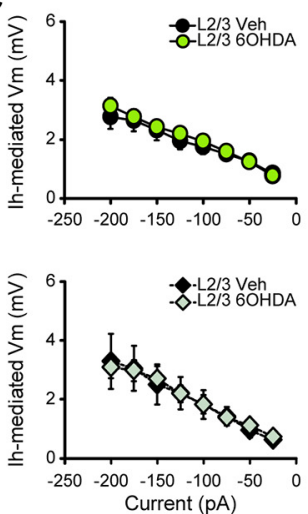

E
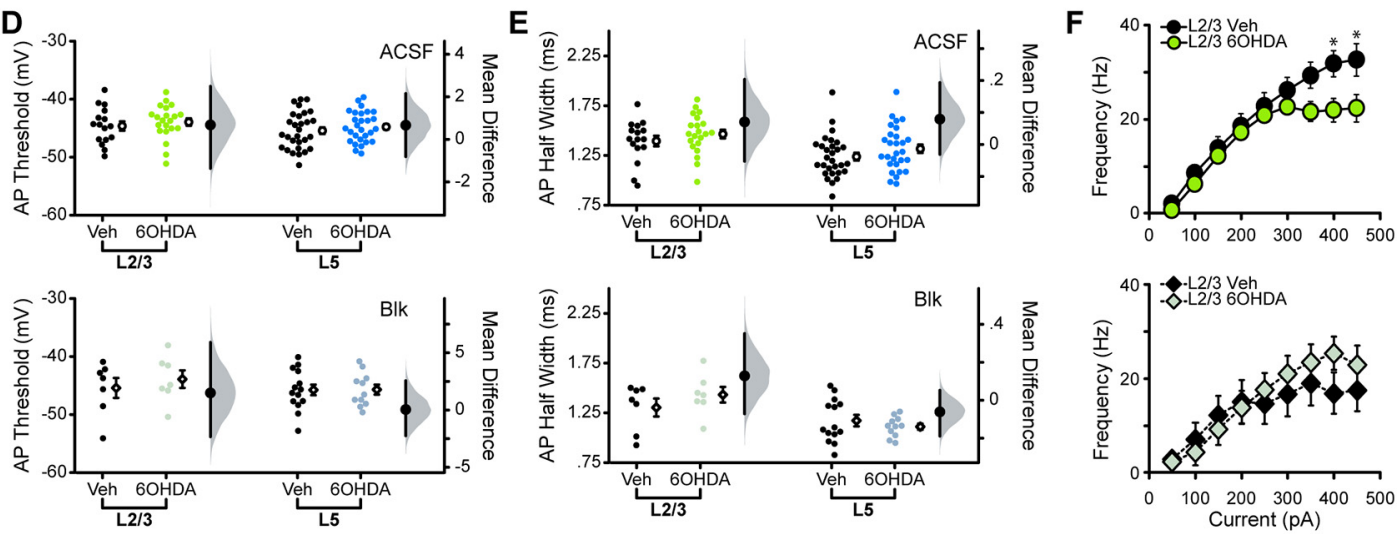

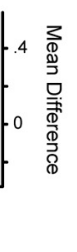

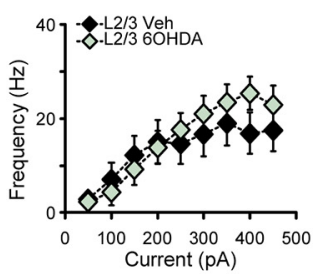
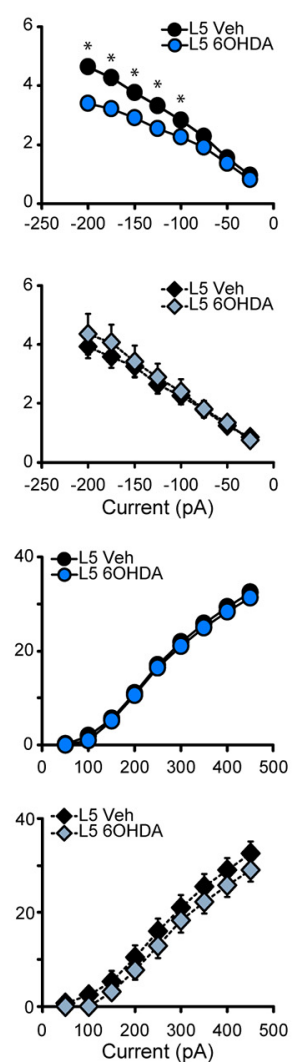

Figure 6. Nigral 6OHDA lesion shifts M1 neuron excitability, partially recapitulating the effects of D1R antagonist. $\boldsymbol{A}$, Superimposed responses to hyperpolarizing and depolarizing current steps in individual L2/3 (green) and L5 (blue) neurons of vehicle- and 6OHDAinjected animals. All traces shown are in ACSF conditions. Scale bar: Top, $20 \mathrm{mV}, 100 \mathrm{~ms}$; bottom, $10 \mathrm{mV}, 100 \mathrm{~ms}$. B-F, Summary excitability plots for excitatory neurons in L2/3 and L5 of vehicle and 6OHDA animals, in baseline (ACSF) or synaptic blockers (BLK; $20 \mu \mathrm{m}$ picrotoxin, $20 \mu \mathrm{m}$ DNQX, $50 \mu \mathrm{m}$ AP5) conditions. Modified Cumming plots show raw data of individual neurons as swarm plots, with the mean \pm SEM offset to the right. Further right of each group of raw data are the effect size (black circle), corresponding $95 \% \mathrm{Cls}$ (black vertical bars), and the underlying bootstrap sampling distribution. B. Dynamic input resistance across hyperpolarizing current steps. C, Voltage dependence of $I_{\mathrm{h}}$-mediated voltage sag elicited by hyperpolarizing current. $\boldsymbol{D}$, Action potential threshold at rheobase. $\boldsymbol{E}$, Action potential half-width at rheobase. $\boldsymbol{F}$, Action potential frequency during suprathreshold current injections. ACSF L2/3 vehicle neurons: $N=11, n=16$; ACSF L2/3 6OHDA neurons: $N=13, n=21$; BLK L2/3 vehicle neurons: $N=3, n=7$; BLK L2/3 6OHDA neurons: $N=4, n=8$; ACSF L5 vehicle neurons: $N=11, n=29$; ACSF L5 6OHDA neurons: $N=11, n=29$; BLK L5 vehicle neurons: $N=5, n=14$; BLK L5 6OHDA neurons: $N=6, n=12$. Data are shown as the mean \pm SEM. ${ }^{*} p \leq 0.05$.

contralateral to the injection, while vehicle-injected animals showed no sign of forelimb use impairment (Fig. $5 C$; vehicle, $0.52 \pm 0.017 ; 6 \mathrm{OHDA}, 0.25 \pm 0.032 ; p=3.58 \times$ $\left.10^{-8}\right)$. The severity of the 6OHDA lesion was anatomically assessed with post hoc immunostaining for $\mathrm{TH}$, followed by unbiased stereological counts of $\mathrm{TH}^{+}$neurons in the VTA and SNc. Lesioned animals showed significant cell loss in both the SNc and VTA when compared with their vehicle-injected counterparts [Fig. $5 D, E ; \mathrm{TH}^{+}$neuron ratio: vehicle SNc, $0.79 \pm 0.049$; $6 \mathrm{OHDA} \mathrm{SNc}, 0.037 \pm$ $0.015\left(p=1.31 \times 10^{-14}\right)$; vehicle VTA, $1.01 \pm 0.063 ; 60 H D A$ VTA, $\left.0.30 \pm 0.048\left(p=3.32 \times 10^{-9}\right)\right]$, indicating that the unilateral injection had effectively depleted dopamine neurons and induced the expected motor impairment. Because the 6OHDA lesion caused a significant reduction of neurons in the VTA, we posited that this could lead to a reduction in $\mathrm{TH}^{+}$boutons in $\mathrm{M} 1$, since $\mathrm{M} 1$ is a target of dopaminergic projections from the VTA and, to a lesser extent, the SNc (Hosp et al., 2011; Leemburg et al., 2018). To test this prediction, we performed stereological counts of $\mathrm{TH}^{+}$boutons in L2/3 and L5 in a subset of lesioned and vehicle-injected animals where sections containing $\mathrm{M} 1$ had been collected and processed. Unilateral 6OHDA lesion of the $\mathrm{SNc}$ led to significant reduction of $\mathrm{TH}^{+}$boutons in the ipsilateral M1 (Fig. 5F; vehicle L2/3, $1.14 \pm 0.058$; 6OHDA L2/3, $0.29 \pm 0.034(p=0.0063)$; vehicle L5, $1.02 \pm 0.067 ; 6 \mathrm{OHDA}$ $\mathrm{L} 5,0.31 \pm 0.12(p=0.035)]$. These data indicate that a nigral 6OHDA lesion is sufficient to reduce both midbrain dopaminergic cells as well as direct dopaminergic innervation of M1.

We then assessed the effect of the 6OHDA manipulation on the input/output function of $L 2 / 3$ and $L 5$ pyramidal neurons in M1 (Fig. 6). In contrast to the acute blockade of D1R and D2R, chronic midbrain dopamine depletion did not alter input resistance, action potential threshold, or action potential half-width of either L2/3 or L5 neurons in $\mathrm{M} 1$ (Fig. $6 B, D, E$ ). Interestingly, the voltage sag was unaffected in L2/3 neurons, but significantly reduced in L5 
A

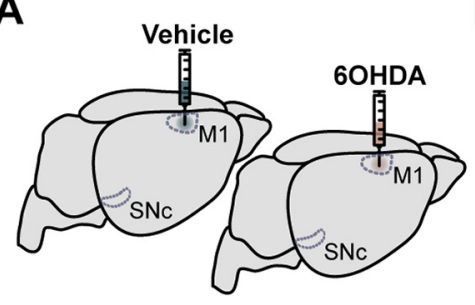

B

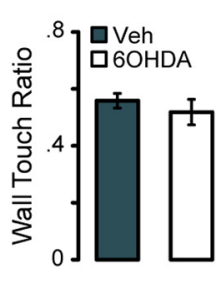

C

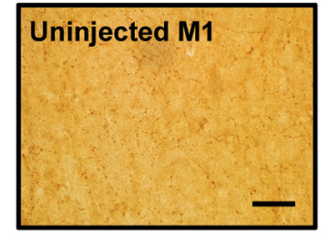

G

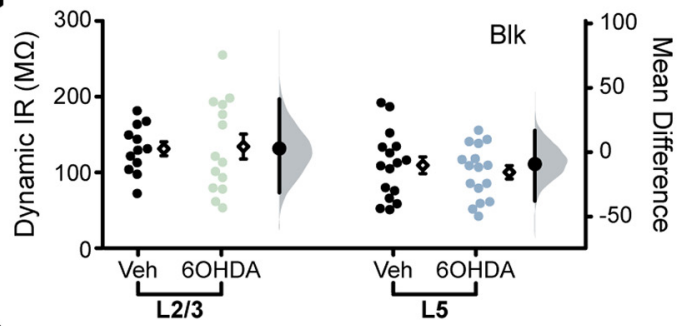

D

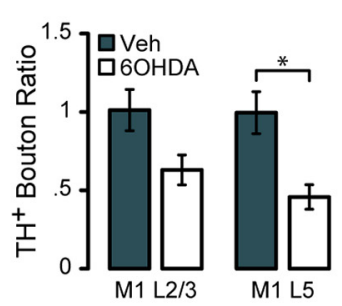

F

Layer 2/3

Blk M1 Vehicle

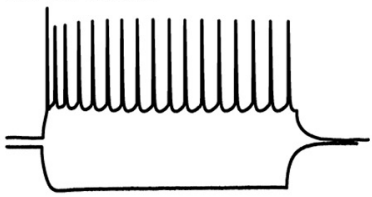

Layer 5

Blk M1 Vehicle

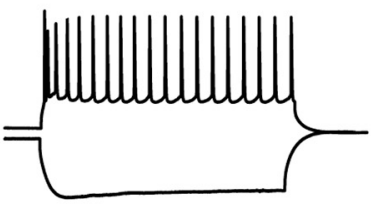

E

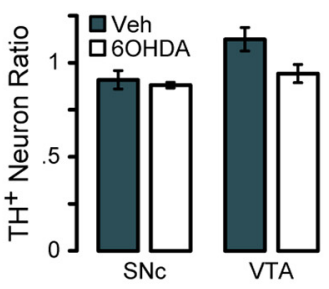

H

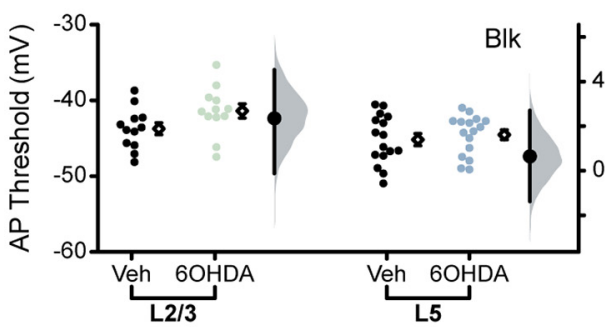

I

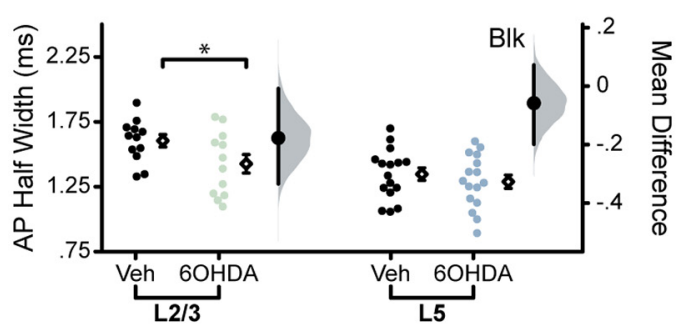

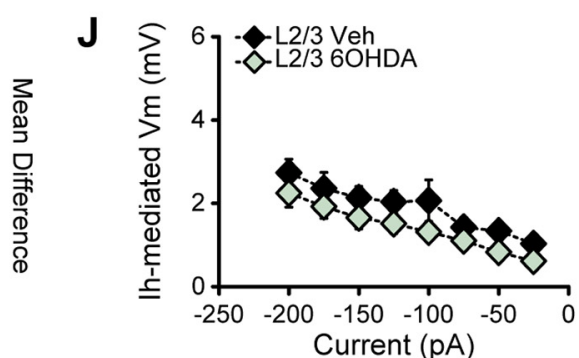

$\mathrm{K}$

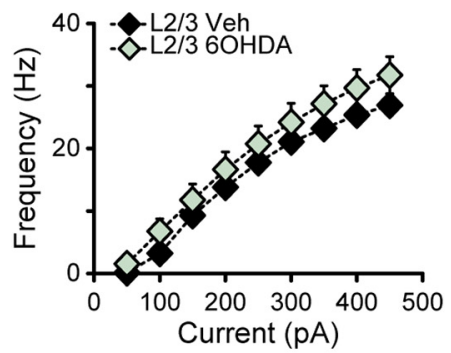

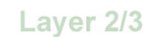

Blk M1 6OHDA

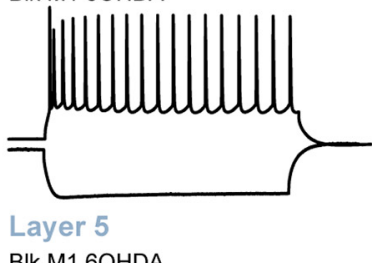

Blk M1 6OHDA

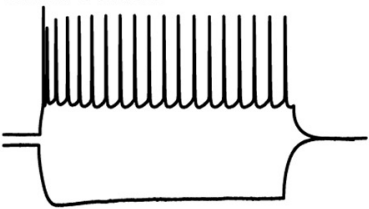

Figure 7. Chronic M1 dopamine depletion impacts $L 2 / 3$ intrinsic excitability. A, Unilateral injection of $60 H D A$ or vehicle into forelimb region of $\mathrm{M} 1$. B, Quantification of weight-bearing wall touches in cylinder motor assessment. $\boldsymbol{C}, \mathrm{TH}^{+}$axons and boutons labeled in L2/3 of M1 ipsilateral and contralateral to the injection site. Magnification, $40 \times$. Scale bar, $50 \mu \mathrm{m}$. $\boldsymbol{D}$, Stereological counts of TH ${ }^{+}$ boutons in $\mathrm{M} 1$ in lesioned $(N=4)$ or vehicle-injected $(N=5)$ animals. $\boldsymbol{E}$, Stereological counts of TH ${ }^{+}$neurons in the SNc and VTA of a subset of animals (vehicle, $N=4$; $6 \mathrm{OHDA}, N=4$ ). $\boldsymbol{F}-\boldsymbol{K}$, Summary excitability plots for excitatory neurons in L2/3 (green) and L5 (blue) of vehicle or 6OHDA-injected animals, performed in synaptic blockers (BLK: $20 \mu \mathrm{m}$ picrotoxin, $20 \mu \mathrm{M}$ DNQX, 50 $\mu \mathrm{M}$ AP5). Modified Cumming plots show raw data of individual neurons as swarm plots, with the mean \pm SEM offset to the right. Further right of each group of raw data are the effect size (black circle), corresponding 95\% Cls (black vertical bars), and the underlying bootstrap sampling distribution. $\boldsymbol{F}$, Superimposed responses to hyperpolarizing and depolarizing current steps in individual $L 2 / 3$ and $L 5$ neurons in vehicle- or 6OHDA-injected animals. Scale bar: Top, $20 \mathrm{mV}, 100 \mathrm{~ms}$; bottom, $10 \mathrm{mV}, 100 \mathrm{~ms}$. G, Dynamic input resistance across hyperpolarizing current steps. $\boldsymbol{H}$, Action potential threshold at rheobase. I, Action potential half-width at rheobase. $\boldsymbol{J}$, Voltage dependence of $I_{\mathrm{h}}$-mediated voltage sag elicited by hyperpolarizing current. $\boldsymbol{K}$, Action potential frequency during suprathreshold current injections. Vehicle L2/3 neurons: $N=4, n=12$; vehicle L5 neurons: $N=5, n=16$; 6 OHDA L2/3 neurons: $N=4, n=14$; $6 O H D A$ L5 neurons: $N=4, n=16$. Data are shown as the mean \pm SEM. ${ }^{*} p \leq 0.05$. 
(Fig. 6C). As acute D1R antagonism in L5 drove an increase in voltage sag, this indicates that the ablation of midbrain dopamine neurons engages mechanisms impacting M1 excitability that go beyond reduced activity of D1R. Further, this effect was eliminated when the experiment was performed in synaptic blockers, pointing to the involvement of synaptic mechanisms in the effect of chronic nigral dopamine depletion. These results were reinforced by estimation statistics performed on the data (Tables 1, 2), which together suggest that chronic loss of dopaminergic activity in the motor circuit does not fully recapitulate the effects of acute dopamine receptor blockade in $\mathrm{M} 1$.

Analysis of $f-l$ curves for both $L 2 / 3$ and $L 5$ pyramidal neurons unveiled an effect similar to the effect of acute D1R blockade: $L 2 / 3$ neurons of 6 OHDA mice lost the ability to increase their firing rate at the highest current injections (Fig. $6 F$ ), and this effect was abolished by the application of synaptic blockers. The $f-I$ curve of L5 neurons in 6OHDA mice was not significantly different from that of vehicle-injected mice in either ACSF or synaptic blockers (Fig. 6F). These results suggest that midbrain depletion of dopamine induces laminar-specific changes in the excitability of M1 pyramidal neurons, likely through a combination of altered dopaminergic signaling in $\mathrm{M} 1$ as well as through a shift in overall synaptic transmission in the area.

\section{Chronic depletion of dopaminergic input to M1 exclusively impacts $L 2 / 3$ excitability}

The nigral 6OHDA lesion primarily affected the SNc but also reduced the number of dopaminergic neurons in the VTA and $\mathrm{TH}^{+}$boutons in M1. The effects of this manipulation only partially overlapped with those of acute dopamine receptors antagonism, leading us to investigate how the depletion of dopaminergic afferents exclusively in M1 would impact excitatory neuron excitability. Unilateral 6OHDA lesion of M1 did not induce motor impairment in the cylinder motor task compared with vehicle-injected controls [Fig. 7B; vehicle, $0.56 \pm 0.026 ; \quad 60 \mathrm{HDA}, 0.52 \pm 0.045$ $(p=0.49)]$. Stereological counts of $\mathrm{TH}^{+}$boutons in $\mathrm{M} 1$ showed that the 6OHDA lesion significantly reduced $\mathrm{TH}^{+}$ boutons in ipsilateral L5 and reduction trending toward significance in ipsilateral L2/3 [Fig. 7C,D; vehicle L2/ $3,1.012 \pm 0.13$; 6 OHDA L2/3, $0.63 \pm 0.10(p=0.068)$; vehicle L5, $1.00 \pm 0.13$; 6OHDA L5, $0.46 \pm 0.079(p=0.017)$ ], indicating that the 6OHDA injection was effective. In a subset of animals where midbrain tissue had been collected and processed, we performed stereological counts of $\mathrm{TH}^{+}$neurons in the SNc and VTA to assess whether a 6OHDA lesion of $\mathrm{M} 1$ was sufficient to reduce dopaminergic neurons in the midbrain. Unilateral M1 6OHDA lesion had no impact on $\mathrm{TH}^{+}$neurons in the ipsilateral SNc, while counts in the ipsilateral VTA showed a reduction in $\mathrm{TH}^{+}$neurons that was trending toward significance [Fig. 7E; M1 vehicle SNc, $0.91 \pm 0.070 ;$ M1 6OHDA SNc, $0.88 \pm 0.052(p=0.76)$; M1 vehicle VTA, $1.12 \pm 0.030 ;$ M1 6OHDA VTA, $0.94 \pm 0.073$ $(p=0.059)]$.

As the effects of acute D1R and D2R blockade were largely independent of synaptic transmission, patchclamp recordings in M1 lesion experiments were conducted in the presence of synaptic blockers. L5 pyramidal neurons showed no differences in the subthreshold or suprathreshold range of activity (Fig. $7 F-K$, Table 2), suggesting that chronic loss of dopaminergic input to M1 did not affect the excitability of these neurons. In contrast, L2/3 neurons showed several changes following 6OHDA lesion in M1. First, the action potential threshold was depolarized, and statistical analysis of these data revealed that this effect was trending toward significance [Fig. $7 H$, Table 1; in $\mathrm{mV}$ : L2/3 vehicle, $-43.74 \pm 0.79$; L2/3 6 OHDA, $-41.39 \pm 0.93(p=0.067)]$, indicating that larger currents are required for these neurons to generate an action potential. Second, the action potential half-width of L2/3 neurons was significantly reduced following 6OHDA M1 lesioning [Fig. 7I, Table 1; in ms: L2/3 vehicle, $1.60 \pm$ 0.048 ; L2/3 6OHDA, $1.43 \pm 0.071(p=0.050)]$. These shifts in L2/3 neuron excitability are specific to the $M 1$ lesion experiment and are not recapitulated by either dopamine receptor blockade or midbrain dopaminergic cell loss. This suggests that local dopaminergic deafferentation within M1 causes unique changes in excitability specific to L2/3 pyramidal neurons. Together, our results indicate that dopamine impairment can have complex effects on the input/output function of M1 neurons depending on the duration and location of the dopamine impairment.

\section{Discussion}

Dopaminergic signaling is crucial for skilled voluntary movement, and reduced dopamine in the motor circuit leads to motor impairment. PD is characterized by progressive dopaminergic cell death in the SNc, which primarily projects to the basal ganglia, and a less severe but significant loss of dopaminergic neurons in the VTA (Giguère et al., 2018). While the effects of reduced dopamine signaling has been well documented in the basal ganglia (Blandini et al., 2000; Day et al., 2006; Azdad et al., 2009; Bagetta et al., 2010; Fieblinger et al., 2014), how midbrain dopaminergic cell death affects $\mathrm{M} 1$ is less clear. Previous studies have highlighted the importance of dopamine in M1 for motor learning and plasticity (MolinaLuna et al., 2009; Rioult-Pedotti et al., 2015), and impairment of dopaminergic input to M1 in vivo results in impaired skill learning, delayed movement execution, and structural changes at M1 synapses (Hosp et al., 2011; Guo et al., 2015; Chen et al., 2019); however, the mechanisms underlying these changes are understudied. We demonstrate that impaired dopamine signaling impacts the excitability of M1 pyramidal neurons, an effect that could contribute to the diminished motor function observed in previous studies.

Acute D1R and D2R antagonism impacts the input/output function of $M 1$ neurons, suggesting that dopamine regulates excitability in a healthy $\mathrm{M} 1$. Our results show that D1R blockade increases the excitability of L2/3 and L5 neurons in the subthreshold range of activity up to rheobase. Within the suprathreshold range of activity, on the other hand, layer-specific effects emerge. The $f-I$ curve of L2/3 neurons was shifted downward following D1R antagonism, particularly impacting the response to large input. This effect could be driven by a depolarization 
block, which has been observed in hippocampal pyramidal neurons (Bianchi et al., 2012) and midbrain dopaminergic neurons (Canavier et al., 2016). In these instances, depolarization blocks have been ascribed to changes in kinetics and/or conductance of voltage-gated sodium channels (Qian et al., 2014) and delayed rectifier potassium channels (Bianchi et al., 2012). Other modeling studies investigating the currents that govern firing frequency and gain attribute the downshift to increased sodium conductance (Kispersky et al., 2012). In contrast, L5 neurons showed an upward shift in the linear portion of the $f-I$ curve when fast synaptic transmission was blocked. This implies that D1R antagonism caused increased excitability of L5 neurons in their suprathreshold range as well; however, this effect was masked when spontaneous synaptic transmission was present. In fact, almost all effects of D1R antagonism persisted in the presence of fast synaptic transmission blockers, consistent with the direct expression of these receptors on the cells we recorded and with the engagement of intrinsic mechanisms for the modulation of membrane properties. The effects of D2R blockade were more subtle, although consistent with an overall increase in excitability in L2/3 and L5. These changes appear to be dependent on synaptic activity in L2/3, where the effects of D2R blockade were eliminated by synaptic transmission blockers. In contrast, many of the excitability shifts in L5 following D2R blockade, including an upward shift in the linear portion of the L5 $\mathrm{f}-\mathrm{I}$ curve, persisted when synaptic transmission was blocked. The layer-specific differences in excitability following dopamine receptor blockade, particularly those that are synaptic transmission dependent, could be because of a differential and cell type-specific distribution of $\mathrm{D} 1 \mathrm{R}$ and D2R expression. Effects that were abolished when synaptic transmission was blocked could be the result of D1R/D2R antagonism on other neurons, including inhibitory neurons, an effect that may shift incoming excitation or inhibition onto the recorded neurons in a manner that influences their excitability. Studies have shown that cortical inhibitory interneurons differentially express D1R and D2R subtypes (Le Moine and Gaspar, 1998; Anastasiades et al., 2019) and that increasing or decreasing their activity via dopaminergic modulation could in turn shift the excitability of neighboring pyramidal cells. Further studies are needed to examine the consequences of reduced dopamine signaling on interneurons in M1 and to understand their interaction with excitatory neurons in the same layer.

The increase in excitability following D1R blockade is surprising in view of studies in the basal ganglia showing that dopamine or D1R agonists typically increase excitability (Planert et al., 2013). However, reports show that dopamine, via D1R, reduces the excitability of L5 pyramidal neurons in the entorhinal cortex (Rosenkranz and Johnston, 2006). Additionally, dopamine application in M1 in vivo reduces spontaneous firing of corticospinal neurons (Huda et al., 2001; Awenowicz and Porter, 2002), which are most densely found in M1 L5 (Oswald et al., 2013). Analysis of neuronal excitability in experiments in which slices were preincubated in dopamine, and then a cocktail of dopamine and D1R and D2R antagonists, mimicked a transient shutdown of dopaminergic activity in M1 and induced shifts in excitability in accordance with these studies.

After establishing that the excitability of M1 pyramidal neurons is directly modulated by dopamine, we extended our study to assess how chronic loss of dopamine modulates the excitability of M1 pyramidal neurons. Loss of dopaminergic neurons projecting to the motor circuit, particularly to the basal ganglia, leads to movement dysfunction and is a hallmark of PD. Chronic depletion of dopamine impacts neural activity across the motor circuit (Blandini et al., 2000; Day et al., 2006; Azdad et al., 2009; Planert et al., 2013; Benazzouz et al., 2014). We posited that chronic loss of dopamine to all areas involved in motor control would lead to reverberating changes in excitability within $\mathrm{M} 1$, despite this region receiving only a fraction of the dopaminergic input. In agreement with previous work, our $6 \mathrm{OHDA}$-injected mice showed unilaterally impaired forelimb use. Furthermore, 6OHDA mice showed laminar-specific shifts in excitability within $\mathrm{M} 1$, indicating that neurons in L2/3 and L5 have distinct sensitivity to chronic loss of dopamine signaling. Contrasting the effects of acute D1R blockade, L2/3 neurons in 6OHDA mice showed no change in the subthreshold range of activity. However, the $f-l$ curve of L2/3 neurons showed impaired response to large inputs, mirroring the effect of acute D1R antagonism. This change was eliminated by synaptic transmission blockers, suggesting that, unlike the effect of acute D1R blockade, this comparable shift in the $f-I$ curve of $L 2 / 3$ neurons is dependent on synaptic transmission. The excitability of L5 neurons was also affected by chronic dopamine depletion, although the effects were unique to this cortical layer and dopamine manipulation. Chronic midbrain dopamine depletion induced a decrease in $I_{\mathrm{h}}$ of L5 cells with no changes in the suprathreshold range of their input/output function. The decrease in $I_{\mathrm{h}}$ was fully eliminated by the application of fast synaptic transmission blockers, suggesting that it is mediated by synaptic activity. Decreased $I_{\mathrm{h}}$ alters the capacity of the neuron to maintain resting membrane potential and impacts the response to input, particularly those that would hyperpolarize the cell.

Overall, the changes in excitability because of midbrain dopamine loss rely on synaptic drive, suggesting that chronic midbrain dopamine manipulation may alter fast synaptic transmission into or within M1. This could occur as a consequence of shifting activity within the basal ganglia following dopamine loss, as an effect of reduced dopamine signaling directly in $\mathrm{M} 1$, or a combination of these factors. While a thorough analysis of synaptic transmission is beyond the scope of this study, there are reports of altered synaptic activity in models of PD elsewhere in the motor circuit (Day et al., 2006; Fan et al., 2012; Galvan et al., 2015; Parker et al., 2016), pointing to the possibility that the excitatory drive of $\mathrm{M} 1$ may be reduced.

As the effects of a nigral 6OHDA lesion only partially overlapped with those observed using dopamine receptor antagonists, we predicted that these changes to M1 pyramidal neuron input/output function were likely because 
of altered synaptic activity across the motor circuit rather than simply from the loss of dopaminergic input exclusively to M1. In support of this interpretation, restricting the 6OHDA injection to M1 altered the excitability of L2/3, but not L5, pyramidal neurons with apparently opposing effects to those caused by midbrain dopamine depletion or acute D1R and D2R blockade. The action potential threshold of $\mathrm{L} 2 / 3$ pyramidal neurons was depolarized and the half-width was decreased, indicating a shift in the currents governing action potential dynamics around rheobase. These results differ from those obtained following all other manipulations. We speculate that they result from a combinatorial, chronic reduction in M1 D1R and D2R activity on all neuron types and may result from compensatory mechanisms following the chronic local loss of dopaminergic innervation. Dopamine depletion in M1 did not induce motor impairment, pointing to a limited impact of direct dopaminergic projections in motor execution. Such a result is expected, as direct dopamine modulation in $\mathrm{M} 1$ is primarily associated with synaptic plasticity and motor learning, aspects that we did not examine (MolinaLuna et al., 2009; Hosp et al., 2011).

\section{Functional implications}

Dopamine receptor blockade increased the excitability of L2/3 and L5 neurons. In M1, L2/3 pyramidal neurons are mainly corticocortical or corticostriatal projecting, while those in L5 are corticospinal, corticothalamic, and corticostriatal projecting (Oswald et al., 2013). Signals flow superficial to deep, with high intracortical connectivity between L2/3 neurons and corticospinal neurons in L5 (Weiler et al., 2008). Our results show that acute impairment of D1R and D2R signaling increases the excitability of $L 5$ neurons along with the excitability of one of their primary presynaptic partners. Together, these changes may lead to hyperactivity in $\mathrm{M} 1$, an effect associated with motor impairment and movement disorders like PD (Thobois et al., 2000; Haslinger et al., 2001).

Our results also show that acute dopamine receptor antagonism increased the $I_{\mathrm{h}}$-mediated sag in L2/3 and L5, while chronic dopamine loss by midbrain 6OHDA injections decreased the sag selectively in L5. In M1, In is thought to be a regulator of signal flow from superficial layers, which is involved in motor planning, to deeper output layers (Sheets et al., 2011). We show that dopamine can directly and indirectly influence the activity of $I_{\mathrm{h}}$, providing evidence for one source of neuromodulatory control of signal flow in M1. Interestingly, one study of an animal model of PD reported downregulation of HCN2 channels, which in part mediate $I_{\mathrm{h}}$, in the globus pallidus (Chan et al., 2011). This led to abolished autonomous pacemaking activity and induced abnormal synchronous activity. While reintroduction of HCN2 channels in the globus pallidus restored normal signaling, it was not sufficient to recover motor impairments. In view of our findings, one may speculate that abnormal $\mathrm{HCN}$ activity in PD may extend beyond the globus pallidus, and that coordinated restoration of the activity of these channels may be required for symptom improvement.

\section{Conclusions}

Dopamine signaling in the motor system is crucial for the execution of voluntary movements. While most work focuses on the effects of dopamine signaling in the basal ganglia, recent studies point to M1 as an additional site of dysfunction in PD patients and mouse models of the disease. Our results indicate that diminished dopamine signaling, whether acute or chronic, has profound effects on the excitability of M1 neurons. We unveil a complex combination of laminar-specific mechanisms for dopaminedependent modulation of pyramidal neuron excitability, which are likely to significantly alter the output of M1 and influence movement execution.

\section{References}

Albin RL, Young AB, Penney JB (1989) The functional anatomy of basal ganglia disorders. Trends Neurosci 12:366-375.

Anastasiades PG, Boada C, Carter AG (2019) Cell-type-specific D1 dopamine receptor modulation of projection neurons and interneurons in the prefrontal cortex. Cereb Cortex 29:3224-3242.

Ariano MA, Sibley DR (1994) Dopamine receptor distribution in the rat CNS: elucidation using anti-peptide antisera directed against D1A and D3 subtypes. Brain Res 649:95-110.

Awenowicz PW, Porter LL (2002) Local application of dopamine inhibits pyramidal tract neuron activity in the rodent motor cortex. $J$ Neurophysiol 88:3439-3451.

Azdad K, Chàvez M, Don Bischop $P$, Wetzelaer $P$, Marescau $B$, De Deyn PP, Gall D, Schiffmann SN (2009) Homeostatic plasticity of striatal neurons intrinsic excitability following dopamine depletion. PLoS One 4:e6908.

Bagetta V, Ghiglieri V, Sgobio C, Calabresi P, Picconi B (2010) Synaptic dysfunction in Parkinson's disease. Biochem Soc Trans 38:493-497.

Barbas H, García-Cabezas MA (2016) How the prefrontal executive got its stripes. Curr Opin Neurobiol 40:125-134.

Beckstead RM, Domesick VB, Nauta WJ (1979) Efferent connections of the substantia nigra and ventral tegmental area in the rat. Brain Res 175:191-217.

Benazzouz A, Mamad O, Abedi P, Bouali-Benazzouz R, Chetrit J (2014) Involvement of dopamine loss in extrastriatal basal ganglia nuclei in the pathophysiology of Parkinson's disease. Front Aging Neurosci 6:87.

Bianchi D, Marasco A, Limongiello A, Marchetti C, Marie H, Tirozzi B, Migliore M (2012) On the mechanisms underlying the depolarization block in the spiking dynamics of CA1 pyramidal neurons. $J$ Comput Neurosci 33:207-225.

Blandini F, Nappi G, Tassorelli C, Martignoni E (2000) Functional changes of the basal ganglia circuitry in Parkinson's disease. Prog Neurobiol 62:63-88.

Bosch-Bouju C, Hyland BI, Parr-Brownlie LC (2013) Motor thalamus integration of cortical, cerebellar and basal ganglia information: implications for normal and parkinsonian conditions. Front Comput Neurosci 7:163

Calabresi P, Di Filippo M (2015) The changing tree in Parkinson's disease. Nat Neurosci 18:1196-1198.

Calin-Jageman RJ, Cumming G (2019) Estimation for better inference in neuroscience. eNeuro 6:ENEURO.0205-19.2019.

Campbell MJ, Morrison JH (1989) Monoclonal antibody to neurofilament protein (SMI-32) labels a subpopulation of pyramidal neurons in the human and monkey neocortex. J Comp Neurol 282:191205.

Canavier CC, Evans RC, Oster AM, Pissadaki EK, Drion G, Kuznetsov AS, Gutkin BS (2016) Implications of cellular models of dopamine neurons for disease. J Neurophysiol 116:2815-2830.

Chan CS, Glajch KE, Gertler TS, Guzman JN, Mercer JN, Lewis AS, Goldberg AB, Tkatch T, Shigemoto R, Fleming SM, Chetkovich 
DM, Osten P, Kita H, Surmeier DJ (2011) HCN channelopathy in external globus pallidus neurons in models of Parkinson's disease. Nat Neurosci 14:85-92.

Chen K, Vincis R, Fontanini A (2019) Disruption of cortical dopaminergic modulation impairs preparatory activity and delays licking initiation. Cereb Cortex 29:1802-1815.

Day M, Wang Z, Ding J, An X, Ingham CA, Shering AF, Wokosin D, Ilijic E, Sun Z, Sampson AR, Mugnaini E, Deutch AY, Sesack SR, Arbuthnott GW, Surmeier DJ (2006) Selective elimination of glutamatergic synapses on striatopallidal neurons in Parkinson disease models. Nat Neurosci 9:251-259.

Fallon JH, Moore RY (1978) Catecholamine innervation of the basal forebrain. IV. Topography of the dopamine projection to the basal forebrain and neostriatum. J Comp Neurol 180:545-580.

Fan KY, Baufreton J, Surmeier DJ, Chan CS, Bevan MD (2012) Proliferation of external globus pallidus-subthalamic nucleus synapses following degeneration of midbrain dopamine neurons. $J$ Neurosci 32:13718-13728.

Fieblinger T, Graves SM, Sebel LE, Alcacer C, Plotkin JL, Gertler TS, Chan CS, Heiman M, Greengard P, Cenci MA, Surmeier DJ (2014) Cell type-specific plasticity of striatal projection neurons in parkinsonism and L-DOPA-induced dyskinesia. Nat Commun 5:5316.

Fu Y, Yuan Y, Halliday G, Rusznák Z, Watson C, Paxinos G (2012) A cytoarchitectonic and chemoarchitectonic analysis of the dopamine cell groups in the substantia nigra, ventral tegmental area, and retrorubral field in the mouse. Brain Struct Funct 217:591612.

Galvan A, Devergnas A, Wichmann T (2015) Alterations in neuronal activity in basal ganglia-thalamocortical circuits in the parkinsonian state. Front Neuroanat 9:5.

Gaspar P, Duyckaerts C, Alvarez C, Javoy-Agid F, Berger B (1991) Alterations of dopaminergic and noradrenergic innervations in motor cortex in Parkinson's disease. Ann Neurol 30:365-374.

Giguère N, Burke Nanni S, Trudeau LE (2018) On cell loss and selective vulnerability of neuronal populations in Parkinson's disease. Front Neurol 9:455.

Grieb B, von Nicolai C, Engler G, Sharott A, Papageorgiou I , Hamel W, Engel AK, Moll CK (2013) Decomposition of abnormal free locomotor behavior in a rat model of Parkinson's disease. Front Syst Neurosci 7:95.

Gundersen HJ (1986) Stereology of arbitrary particles. A review of unbiased number and size estimators and the presentation of some new ones, in memory of William R. Thompson. J Microsc 143:3-45.

Guo L, Xiong H, Kim J-I, Wu Y-W, Lalchandani RR, Cui Y, Shu Y, Xu T, Ding JB (2015) Dynamic rewiring of neural circuits in the motor cortex in mouse models of Parkinson's disease. Nat Neurosci 18:1299-1309.

Haslinger B, Erhard P, Kämpfe N, Boecker H, Rummeny E, Schwaiger M, Conrad B, Ceballos-Baumann AO (2001) Event-related functional magnetic resonance imaging in Parkinson's disease before and after levodopa. Brain 124:558-570.

Ho J, Tumkaya T, Aryal S, Choi H, Claridge-Chang A (2019) Moving beyond $P$ values: data analysis with estimation graphics. Nat Methods 16:565-566.

Hogan QH, Poroli M (2008) Hyperpolarization-activated current (l(h)) contributes to excitability of primary sensory neurons in rats. Brain Res 1207:102-110.

Hooks BM, Mao T, Gutnisky DA, Yamawaki N, Svoboda K, Shepherd GM (2013) Organization of cortical and thalamic input to pyramidal neurons in mouse motor cortex. J Neurosci 33:748-760.

Hosp JA, Molina-Luna K, Hertler B, Atiemo CO, Luft AR (2009) Dopaminergic modulation of motor maps in rat motor cortex: an in vivo study. Neuroscience 159:692-700.

Hosp JA, Pekanovic A, Rioult-Pedotti MS, Luft AR (2011) Dopaminergic projections from midbrain to primary motor cortex mediate motor skill learning. J Neurosci 31:2481-2487.

Huda K, Salunga TL, Matsunami K (2001) Dopaminergic inhibition of excitatory inputs onto pyramidal tract neurons in cat motor cortex. Neurosci Lett 307:175-178. lancu R, Mohapel P, Brundin P, Paul G (2005) Behavioral characterization of a unilateral 6-OHDA-lesion model of Parkinson's disease in mice. Behav Brain Res 162:1-10.

Jankovic J (2008) Parkinson's disease: clinical features and diagnosis. J Neurol Neurosurg Psychiatry 79:368-376.

Khan ZU, Gutiérrez A, Martín R, Peñafiel A, Rivera A, De La Calle A (1998) Differential regional and cellular distribution of dopamine D2-like receptors: an immunocytochemical study of subtype-specific antibodies in rat and human brain. J Comp Neurol 402:353371.

Kida H, Tsuda $\mathrm{Y}$, Ito $\mathrm{N}$, Yamamoto $\mathrm{Y}$, Owada $\mathrm{Y}$, Kamiya $\mathrm{Y}$, Mitsushima D (2016) Motor training promotes both synaptic and intrinsic plasticity of layer II/III pyramidal neurons in the primary motor cortex. Cereb Cortex 26:3494-3507.

Kispersky TJ, Caplan JS, Marder E (2012) Increase in sodium conductance decreases firing rate and gain in model neurons. J Neurosci 32:10995-11004.

Le Moine C, Gaspar P (1998) Subpopulations of cortical GABAergic interneurons differ by their expression of $D 1$ and $D 2$ dopamine receptor subtypes. Brain Res Mol Brain Res 58:231-236.

Leemburg S, Canonica T, Luft A (2018) Motor skill learning and reward consumption differentially affect VTA activation. Sci Rep 8:687.

Lefaucheur JP (2005) Motor cortex dysfunction revealed by cortical excitability studies in Parkinson's disease: influence of antiparkinsonian treatment and cortical stimulation. Clin Neurophysiol 116:244-253.

Lemberger T, Parlato R, Dassesse D, Westphal M, Casanova E, Turiault M, Tronche F, Schiffmann SN, Schütz G (2007) Expression of Cre recombinase in dopaminoceptive neurons. BMC Neurosci 8:4.

Lemon RN (1993) The G. L. Brown Prize Lecture. Cortical control of the primate hand. Exp Physiol 78:263-301.

Lindenbach D, Bishop C (2013) Critical involvement of the motor cortex in the pathophysiology and treatment of Parkinson's disease. Neurosci Biobehav Rev 37:2737-2750.

McGregor MM, Nelson AB (2019) Circuit mechanisms of Parkinson's disease. Neuron 101:1042-1056.

Mink JW (1996) The basal ganglia: focused selection and inhibition of competing motor programs. Prog Neurobiol 50:381-425.

Molina-Luna K, Pekanovic A, Röhrich S, Hertler B, Schubring-Giese M, Rioult-Pedotti M-S, Luft AR (2009) Dopamine in motor cortex is necessary for skill learning and synaptic plasticity. PLoS One 4: e7082.

Nomura S, Bouhadana M, Morel C, Faure P, Cauli B, Lambolez B, Hepp R (2014) Noradrenalin and dopamine receptors both control CAMP-PKA signaling throughout the cerebral cortex. Front Cell Neurosci 8:247.

Oswald MJ, Tantirigama ML, Sonntag I, Hughes SM, Empson RM (2013) Diversity of layer 5 projection neurons in the mouse motor cortex. Front Cell Neurosci 7:174.

Parker PR, Lalive AL, Kreitzer AC (2016) Pathway-specific remodeling of thalamostriatal synapses in Parkinsonian mice. Neuron 89:734-740.

Paz JT, Mahon S, Tiret P, Genet S, Delord B, Charpier S (2009) Multiple forms of activity-dependent intrinsic plasticity in layer $\mathrm{V}$ cortical neurones in vivo. J Physiol 587:3189-3205.

Planert H, Berger TK, Silberberg G (2013) Membrane properties of striatal direct and indirect pathway neurons in mouse and rat slices and their modulation by dopamine. PLoS One 8:e57054.

Qian K, Yu N, Tucker KR, Levitan ES, Canavier CC (2014) Mathematical analysis of depolarization block mediated by slow inactivation of fast sodium channels in midbrain dopamine neurons. J Neurophysiol 112:2779-2790.

Rioult-Pedotti MS, Pekanovic A, Atiemo CO, Marshall J, Luft AR (2015) Dopamine promotes motor cortex plasticity and motor skill learning via PLC activation. PLoS One 10:e0124986.

Rosenkranz JA, Johnston D (2006) Dopaminergic regulation of neuronal excitability through modulation of $I_{\mathrm{h}}$ in layer $V$ entorhinal cortex. J Neurosci 26:3229-3244. 
Santana N, Mengod G, Artigas F (2009) Quantitative analysis of the expression of dopamine D1 and D2 receptors in pyramidal and GABAergic neurons of the rat prefrontal cortex. Cereb Cortex 19:849-860.

Sheets PL, Suter BA, Kiritani T, Chan CS, Surmeier DJ, Shepherd GM (2011) Corticospinal-specific HCN expression in mouse motor cortex: i (h)-dependent synaptic integration as a candidate microcircuit mechanism involved in motor control. J Neurophysiol 106:2216-2231.

Surmeier DJ, Ding J, Day M, Wang Z, Shen W (2007) D1 and D2 dopamine-receptor modulation of striatal glutamatergic signaling in striatal medium spiny neurons. Trends Neurosci 30:228-235.

Tamagnini F, Scullion S, Brown JT, Randall AD (2014) Low concentrations of the solvent dimethyl sulphoxide alter intrinsic excitability properties of cortical and hippocampal pyramidal cells. PLoS One 9:e92557.

Tennant KA, Adkins DL, Donlan NA, Asay AL, Thomas N, Kleim JA, Jones TA (2011) The organization of the forelimb representation of the C57BL/6 mouse motor cortex as defined by intracortical microstimulation and cytoarchitecture. Cereb Cortex 21:865876.

Thobois S, Dominey P, Decety J, Pollak P, Gregoire MC, Broussolle $E(2000)$ Overactivation of primary motor cortex is asymmetrical in hemiparkinsonian patients. Neuroreport 11:785-789.

Vitrac C, Benoit-Marand M (2017) Monoaminergic modulation of motor cortex function. Front Neural Circuits 11:72.

Vitrac C, Péron S, Frappé I, Fernagut P-O, Jaber M, Gaillard A, Benoit-Marand M (2014) Dopamine control of pyramidal neuron activity in the primary motor cortex via D2 receptors. Front Neural Circuits 8:13.

Voelker CC, Garin N, Taylor JS, Gähwiler BH, Hornung JP, Molnár Z (2004) Selective neurofilament (SMI-32, FNP-7 and N200) expression in subpopulations of layer $\mathrm{V}$ pyramidal neurons in vivo and in vitro. Cereb Cortex 14:1276-1286.

Weiler N, Wood L, Yu J, Solla SA, Shepherd GM (2008) Top-down laminar organization of the excitatory network in motor cortex. Nat Neurosci 11:360-366.

Williams SM, Goldman-Rakic PS (1998) Widespread origin of the primate mesofrontal dopamine system. Cereb Cortex 8:321-345. 